\title{
Molecular Dynamics Flexible Fitting Simulations Identify New Models of the Closed State of the Cystic Fibrosis Transmembrane Conductance Regulator Protein
} Dol:

10.1021/acs.jcim.7b00091

\section{Document Version \\ Accepted author manuscript}

Link to publication record in Manchester Research Explorer

Citation for published version (APA):

Simhaev, L., McCarty, N. A., Ford, R. C., \& Senderowitz, H. (2017). Molecular Dynamics Flexible Fitting Simulations Identify New Models of the Closed State of the Cystic Fibrosis Transmembrane Conductance Regulator Protein. Journal of Chemical Information and Modeling, 57(8), 1932-1946. https://doi.org/10.1021/acs.jcim.7b00091

\section{Published in:}

Journal of Chemical Information and Modeling

\section{Citing this paper}

Please note that where the full-text provided on Manchester Research Explorer is the Author Accepted Manuscript or Proof version this may differ from the final Published version. If citing, it is advised that you check and use the publisher's definitive version.

\section{General rights}

Copyright and moral rights for the publications made accessible in the Research Explorer are retained by the authors and/or other copyright owners and it is a condition of accessing publications that users recognise and abide by the legal requirements associated with these rights.

\section{Takedown policy}

If you believe that this document breaches copyright please refer to the University of Manchester's Takedown Procedures [http://man.ac.uk/04Y6Bo] or contact uml.scholarlycommunications@manchester.ac.uk providing relevant details, so we can investigate your claim.

\section{OPEN ACCESS}


This document is confidential and is proprietary to the American Chemical Society and its authors. Do not copy or disclose without written permission. If you have received this item in error, notify the sender and delete all copies.

\section{Molecular Dynamics Flexible Fitting (MDFF) Simulations Identify New Models of Closed State CFTR}

\begin{tabular}{|r|l|}
\hline Journal: & Journal of Chemical Information and Modeling \\
\hline Manuscript ID & ci-2017-000917.R2 \\
\hline Manuscript Type: & Article \\
\hline Date Submitted by the Author: & $28-J u n-2017$ \\
\hline Complete List of Authors: & $\begin{array}{l}\text { Simhaev, Luba; Bar-Ilan University, Chemistry } \\
\text { McCarty, Nael; Emory University School of Medicine and Children's } \\
\text { Healthcare of Atlanta } \\
\text { Ford, Robert; Faculty of Life Sciences, } \\
\text { Senderowitz, Hanoch; Bar-Ilan University, Chemistry }\end{array}$ \\
\hline
\end{tabular}

\section{SCHOLARONE \\ Manuscripts}




\title{
Molecular Dynamics Flexible Fitting (MDFF) Simulations Identify New Models of Closed State CFTR
}

\author{
Luba Simhaev, ${ }^{1}$ Nael A. McCarty, ${ }^{2}$ Robert C. Ford, ${ }^{3}$ Hanoch Senderowitz ${ }^{1}$ * \\ ${ }^{1}$ Department of Chemistry, Bar Ilan University, Ramat-Gan 5290002, Israel \\ ${ }^{2}$ Division of Pulmonology, Allergy/Immunology, Cystic Fibrosis, and Sleep, Department of \\ Pediatrics, Emory + Children's Center for Cystic Fibrosis and Airways Disease Research, \\ Emory University School of Medicine and Children's Healthcare of Atlanta, 2015 Uppergate \\ Drive, Atlanta, GA 30322 \\ ${ }^{3}$ Faculty of Biology Medicine and Health, University of Manchester, Oxford Rd, Manchester, \\ M13 9PL, UK \\ *Corresponding author, e-mail: hsenderowitz@gmail.com
}

Keywords: Cystic Fibrosis, Molecular Dynamics, Molecular Dynamics Flexible Fitting (MDFF) Simulations, CFTR models, Cryo-Electron Microscopy 


\section{Abstract}

Cystic Fibrosis (CF) is a lethal, genetic disease found in particular in humans of European origin which is caused by mutations in the CFTR chloride channel. The search for CF therapies acting by modulating the impaired function of mutant CFTR will be greatly advanced by high resolution structures of CFTR in different states. To date, two medium resolution EM structures of CFTR are available (one of a distant zebrafish (Danio rerio) CFTR ortholog and one of human CFTR). The two models are nearly identical to one another and both correspond to the inward-facing, NBDs separated, closed state of the channel. In addition, lower resolution structural data are available for human CFTR in an alternative conformation which likely features associated NBDs and thus geometrically resembles the conducting state of the channel. Multiple homology models of human CFTR in multiple states have been developed over the years, yet their correspondence to the existing structural information is unexplored. In this work we use molecular dynamics flexible fitting (MDFF) simulations to refine two previously described CFTR models based on the available cryo-EM map of the human protein. This map was recorded in the absence of ATP and consequently represents closed-state CFTR yet its features likely correspond to a NBDs associated conformation of the protein. Accordingly, the resulting models feature dimerized NBDs yet with no membrane traversing pore. Moreover, the open probability of the new models as deduced from the MDFF trajectories is significantly lower than that deduced from control MD trajectories initiated from the starting models. We propose that the new models correspond to a CFTR conformation which to date was largely unexplored yet one that is relevant to the gating cycle of the protein. In particular this conformation may participate in rapid channel opening and closing through small allosteric movements controlled by nucleotide binding and dissociation events. Analyzing the resulting trajectories (and not only the final models as is usually the case), we demonstrate that the refined models have good stereochemical properties and are also in favorable agreement with multiple experimental data. Moreover, despite different starting points, the final models share many common features. Finally, we propose that the combination of high resolution cryo-EM maps which are currently emerging from multiple labs and MDFF simulations will be of value for the development of yet more reliable CFTR models as well as for the identification of binding sites for CFTR modulators. 


\section{Introduction}

Cystic Fibrosis (CF) is a lethal, inherited disease caused by mutations to the Cystic Fibrosis Transmembrane Conductance Regulator (CFTR) chloride channel. ${ }^{1-2}$ Over 2000 CFTR mutations are reported in the literature and over 280 have so far been confirmed as CF-causing by the CFTR2 team (www.cftr2.org). ${ }^{3}$ The mutations differ in their severity and prevalence yet all compromise the ability of the protein to conduct chloride ions across the membrane of epithelial cells. Low chloride conductance disrupts the liquid-salt balance across the cell membrane and when occurring in the epithelial cells lining the airways leads to the accumulation of a viscous mucus layer. This layer is readily colonized by bacteria ultimately resulting in chronic disease and lung failure. ${ }^{2,4}$

Human CFTR is a 1,480-residue glycoprotein, which belongs to the ABC (ATP-Binding Cassette) transporter superfamily. ${ }^{5-6}$ Yet, in contrast with other members of this group, CFTR is the only $\mathrm{ABC}$ transporter known to function as an ion channel. ${ }^{7-10}$ Despite this functional uniqueness, CFTR architecture is similar to that of other ABC transporters and its structure is comprised of two membrane spanning domains (MSDs) linked to two nucleotide binding domains (NBDs) through four intracellular linkers (ICLs). ${ }^{5,11-12}$ In addition, CFTR features a unique, largely disordered R-region which is located at the sequence level between NBD1 and MSD2 and which needs to be phosphorylated to enable channel gating.

The common hypothesis in CF therapy is that restoring chloride conductance to wild type levels may ameliorate most of the pathologies associated with the disease. Since chloride conductance depends both on the number of CFTR channels at the membrane and on their open probability, two complementary strategies have been envisioned. The first relies on so-called CFTR correctors to bring more CFTR channels to the membrane and the second uses CFTR potentiators to increase the open probability of channels already at the membrane. Both strategies are best followed by the development of small molecules that would directly interact with the protein. ${ }^{13}$ Rational discovery of such compounds is likely to be advanced by high resolution structures of CFTR and by an understanding of its folding and gating mechanisms. 
CFTR is a highly dynamic protein with multiple conformations corresponding to different states along its gating cycle. ${ }^{14}$ Multiple conformations are a common feature of ABC transporters. For example, the maltose transporter MalFGK, the lipid flippase MsbA and the lipid-linked oligosaccharide flippase PglK all have multiple conformations as evident from their respective crystal structures (e.g., PDB codes 3FH6 and 3PUW, 3B5W and 3B5Y, and 5C73 and 5C76, for MalFGK, MsbA, and PglK, respectively). Yet in contrast with other ABC transporters, until very recently, a three-dimensional (3D) structure of any of CFTR's states was unavailable. This situation has changed with the publication of the 3D structure of zebrafish (Danio rerio) CFTR at a medium resolution of $3.7 \AA$ (5UAR) from cryo electron microscopy (cryo-EM) ${ }^{15}$ and with the subsequent release of the coordinates of the human protein (5UAK: $3.9 \AA)^{16}$ obtained with the same experimental technique. The two structures which are highly similar to one another feature an inward-facing conformation of the protein with the NBDs completely de-dimerized, which corresponds to its fully closed state.

In the absence of other structures of CFTR, at resolutions high enough to be useful in structurebased drug discovery multiple models of human CFTR were reported in the literature both for the inward facing and for the outward facing states. The outward facing models ${ }^{14,}$ 17-23 were based primarily on the Sav1866 structure as a template, ${ }^{24}$ although recently the newly solved $\mathrm{ABC}$ transporter structure $\mathrm{McjD}^{25}$ has been used to model this state. ${ }^{26}$ Inward facing models were based either on the bacterial $\mathrm{MsbA}^{27}$ or on the $\mathrm{P}-\mathrm{gp}^{28}$ structures. However, new CFTR models of this state were recently reported, based on the structures of the ABC transporters TM287/288 ${ }^{29}$ and ABCB10. ${ }^{30}$ In contrast with the other inward facing models, which have their NBDs largely apart, the NBDs of the TM287/288-based model are partially associated with contacts between the two domains in the vicinity of the degenerate, catalytically inactive ATP site (i.e., the site formed by the Walker A and B motifs of NBD1 and the signature motif of NBD2). Thus this model may represent an interim state of CFTR between the inward facing and outward facing conformations.

Although being mostly based on the same template (Sav1866), all outward facing models are different from one another. The same holds true for the inward facing models (see Table 1), yet, for this state the discrepancy between the models may be resolved by the new structures. 
Differences between the outward facing models of human CFTR are perhaps not surprising since due to the low sequence similarity between CFTR and its homologs, in particular in the MSDs, different modeling efforts utilized different sequence alignments as starting points for model construction and different model refinement procedures. Importantly, despite their differences, all models were reported to be in agreement with multiple experimental data (cross linking, hydrogen bonds, salt bridges, solvent accessibility) and sometimes the same data were used to validate different models. This suggests that while the overall fold of the models is probably correct, available experimental data cannot provide a high enough resolution to determine accurate conformation (or more likely, a set of conformations) of outward facing human CFTR. Yet this is precisely the level of resolution required for effective structure based drug design applications. This in turn stresses the need for high resolution structures for all states of CFTR, preferably of the human protein, or alternatively for more "discriminative" experimental data.

One source of data which was not yet utilized for modeling CFTR structures in an outward facing conformation is cryo-EM. To date, only a low resolution map of wildtype, human CFTR in this conformation is available ${ }^{31}$ from $2 \mathrm{D}$ crystals although higher resolution maps of G551D CFTR are becoming available. ${ }^{32}$ The resultant 2D crystals density map is anisotropic with resolution of $\sim 9 \AA$ parallel to the crystal plane and $\sim 14 \AA$ perpendicular to the plane because of the missing cone of data. ${ }^{33}$ The paper describing the map also reported distortion of the extracellular side of the transmembrane region in one molecule in the asymmetric unit due to contacts with the carbon support film. Lastly, there is also the possibility that some density within the map was due to bound detergent as discussed by the authors. Despite these reservations, we hypothesized that the data from the $2 \mathrm{D}$ crystals could be used for the refinement of models of human CFTR using molecular dynamics flexible fitting (MDFF) simulations. ${ }^{34}$ In order to facilitate the approach and mitigate the effect of potential inaccuracies (see above), we averaged over the two molecules in the unit cell using a real-space correlation search.

In this study we therefore present the refinement of two previously reported homology models of CFTR based on the available EM map of the human protein derived from 2D crystals. The 2D crystals were obtained in the absence of ATP and consequently represent closed state CFTR, yet 
the structure likely corresponds to an outward facing conformation where the two NBDs form a head to tail dimer. In these respects, the map largely differs from the EM maps reported by Zhang and Chen and corresponds to a different state of the protein, one which resembles the conducting state of the channel (but see below). For this reason we chose as starting points for MDFF simulations models that were generated based on the outward facing Sav1866 structure, namely the models reported by Dalton et al. ${ }^{20}$ and by Mornon et al. ${ }^{23}$ While being based on the same template, the two models differ in the initial sequence alignment and the refinement procedure. Thus, Dalton et al. first identified the approximate boundaries of the TMs using heuristic algorithms and then utilized phylogenetic and hydrophobicity analyses to produce a sequence alignment between Sav1866 (template) and CFTR (target). The subsequent homology modeling used this alignment to model most of CFTR yet NBD1 was modeled after its crystal structure (2PZE). Modeling was helped by distance constraints derived from experimentally proposed salt bridges and cysteine cross-linking data. In contrast, Mornon et al. used a combination of sequence alignment tools and hydrophobicity analysis to produce the initial, Sav1866-based sequence alignment with no experimentally derived constraints imposed during model construction. These methodological differences led to different sequence alignments in the $\mathrm{N}$ terminal helix, TM3, TM7, TM8 and TM11 which were translated into a backbone RMSD difference of $5.6 \AA$ between the two final models.

In the present work, each starting model was subjected to multiple MDFF simulations. The simulations were conducted in the absence of ATP to match the experimental conditions under which the map was recorded. To ensure that the refined models are indeed the consequence of the constraints imposed by the electron density, control simulations were performed in the absence of ATP but without the map-derived constraints. In addition, following the refinement procedure, we have removed the map constraints and continued all simulations for an additional 20 ns. Finally, in contrast with most other previous reports, in this work we analyzed entire trajectories rather than final models only.

The final MDFF-refined models were found to correspond to closed-state conformations with no continuous channel traversing the protein yet with the NBDs remaining closely associated (as in the initial models). These closed state models are therefore different from the ATP-free inward- 
facing structures of zebrafish and human CFTR and other models of human CFTR reported to date. Interestingly, these models demonstrate a degree of asymmetry between the N- and Cterminal halves higher than that observed in other outward-facing CFTR models in agreement with the hetero-dimeric nature of the channel. Both the final models as well as the MDFF trajectories were found to be in agreement with multiple experimental data features including cross linking, salt bridges, and probe accessibility. Finally, the trajectories obtained under the EM constraints show a higher percentage of closed conformations than those obtained by the control simulations or the continuation simulations. We propose that these new CFTR models are relevant to the gating cycle of the channel potentially representing a transition state during channel gating, or, an intermediate closed state

\section{Methods}

\subsection{System Preparation}

A map that combined data for the two molecules in the unit cell from the 2D crystals of CFTR (EMD-1966) was generated in Chimera using a real-space correlation search between two copies of the map (Chimera fit-in-map routine) followed by map summation and map cropping. The initial search was guided by the positions of the existing fitted models (PDBID 4a82).

Molecular dynamics (MD) and MDFF simulations ${ }^{34}$ were performed for the human CFTR models in the outward-facing conformation as reported by Dalton et al. ${ }^{20}$ and by Mornon et al. ${ }^{23}$ Prior to simulations, the ATP molecules were removed from the models (since the EM map was recorded in the absence of ATP) and the structures were prepared using the Prepare Protein protocol as implemented in Discovery Studio version 3.5. ${ }^{35}$ This protocol sets the protonation states of titratable residues based on predicted $\mathrm{pKa}$ values. The membrane spanning domains of each prepared model were inserted into an explicit $140 \AA$ X $140 \AA$ membrane patch composed of POPC lipids. The initial positioning of the helices within the membrane was determined using the OPM server. ${ }^{36}$ Then the systems were solvated in TIP3P ${ }^{37}$ water molecules using the SOLVATE package in $\mathrm{VMD}^{38}$ to form a $144 \AA$ X $144 \AA$ X $160 \AA$ and a $144 \AA$ X $144 \AA$ X $158 \AA$ simulation box for the Dalton and Mornon models, respectively. Sodium and chloride ions were added to the water phase using the Ionize package in VMD, in order to neutralize the systems 
and to obtain a salt concentration of $0.15 \mathrm{M}$. Altogether, the systems contained 283,288 atoms and 279,529 atoms for the Dalton and Mornon models, respectively.

\subsection{Molecular Dynamics Flexible Fitting (MDFF) Simulations}

MDFF simulations were performed with NAMD $2.8^{39}$ using the CHARMM36 force field ${ }^{40}$ including the $\varphi / \psi$ cross-term map (CMAP) correction under a Langevin temperature and pressure control, using periodic boundary conditions with particle-mesh Ewald (PME) ${ }^{41}$ electrostatics. First, the simulated systems were energy minimized to remove van der Waals clashes within the system. Following minimization, the systems were equilibrated in three cycles as follows: (1) the lipid membrane tails were allowed to melt for $1 \mathrm{~ns}$, while all other components of the system were fixed in order to introduce the suitable disorder of a fluid-like bilayer; (2) The entire system was allowed to equilibrate for $1 \mathrm{~ns}$ while constraining the positions of the protein atoms; (3) The entire system was allowed to equilibrate for 4 ns with no constraints. Following equilibration, the entire system was rigidly aligned to the EM map using the Situs package ${ }^{42}$ based on the coordinates of the protein. Finally, MDFF production simulations were carried out with the CHARMM36 force field, augmented by the external potentials $U_{\mathrm{EM}}$ (which is derived from the EM map) and $\mathrm{U}_{\mathrm{SS}}$ (which imposes harmonic restraints to maintain the integrity of secondary structure elements (e.g., alpha helices and beta sheets) and to prevent over fitting), as described in Trabuco et al. ${ }^{34}$ Only the protein atoms were coupled to the additional MDFF potentials, while the rest of the system was simulated under the standard force field potential. Different MDFF protocols for the minimization stage using different scaling factors $(1,3,5,7$ and $10 \mathrm{kcal})$, which specify the strength of the effect of the EM map on the system, were examined to obtain the highest correlation coefficient with the map while minimizing over-fitting (see Figure S1 in the Supporting Information). The selected MDFF production phase protocol consisted of three steps as follows: (1) 2 ns of simulation with a scaling factor of $0.3 \mathrm{kcal}$ and with restraints on the dihedral angles of the entire protein with a force constant of $200 \mathrm{kcal} \mathrm{mol}^{-1} \AA^{-2}$; (2) $20 \mathrm{~ns}$ of simulation with a scaling factor of $0.3 \mathrm{kcal}$ while restraining only the secondary structure elements of the protein with a force constant of $200 \mathrm{kcal} \mathrm{mol}^{-1} \AA^{-2}$; (3) $10^{6}$ minimization steps with a scaling factor of $5.0 \mathrm{kcal}$ and with restraints set as in stage 2. During all stages, a constant temperature of $300 \mathrm{~K}$ and a constant pressure of 1 atm. were maintained, and an integration time step of 1 fs was used. Each of the models 
considered in this work was simulated three times, each time starting from a different random seed.

\subsection{Molecular Dynamics Simulations following the MDFF protocol}

Following MDFF simulations the resulting structures from each repeat were subjected to standard molecular dynamic simulations (a total of six simulations). The simulations consisted of two stages: (1) $5 \mathrm{~ns}$ of simulation with restraints on secondary structure elements as applied during the MDFF simulations; (2) 15 ns of simulation with no restraints. During both stages, a constant temperature of $300 \mathrm{~K}$ and a constant pressure of 1 atm. were maintained, and an integration time step of 2 fs was used.

\subsection{Control Dynamic Simulations}

Two control simulations (in the absence of ATP) for each of the models (altogether four simulations) were performed with no application of the $U_{\mathrm{EM}}$ and $\mathrm{U}_{\mathrm{SS}}$ potentials. For these simulations, the systems were minimized and equilibrated according to the protocol described in section 2.2, and then simulated for $20 \mathrm{~ns}$ with a constant temperature of $300 \mathrm{~K}$, a constant pressure of 1 atm., and an integration time step of $2 \mathrm{fs}$.

\subsection{Data Analysis}

The final structures from each simulation were tested first for their fit to the EM map and their stereochemical quality, then analyzed for the existence of a membrane traversing pore, and finally evaluated for their agreement with available experimental data (e.g. salt bridges, hydrogen bonds, cross linking data, solvent accessibility). Similar parameters were also evaluated for the complete trajectories. All calculations were performed using the trajectory analysis tools in VMD except as noted below.

The stereochemical integrity of the resulting models was evaluated using Ramachandran plots calculated with Discovery Studio version 3.5. Fitting to the EM maps was evaluated by calculating cross correlation coefficients (CC) using equation (1):

$C C=\frac{\langle(S-\langle S\rangle)(E-\langle E\rangle)\rangle}{\sigma_{S} \sigma_{E}}$ 
Where $<\mathrm{S}>$ and $<\mathrm{E}>$ are the average voxel values of the simulated and experimental density maps, respectively, and $\sigma_{\mathrm{S}}$ and $\sigma_{\mathrm{E}}$ are their standard deviations.

$\mathrm{CC}$ values for complete trajectories were calculated with $\mathrm{VMD}^{38}$ and those for the initial and final models were also calculated with Chimera. ${ }^{43}$ Both Chimera and VMD use equation (1) and the differences between the final values (those of Chimera being higher) likely emerge from the usage of different map threshold values during the fitting process. Chimera values are given to allow for the reproduction of the results using this popular software.

Solvent accessible surface area (SASA) analysis was performed using Discovery Studio version 3.5. ${ }^{35}$ The analysis was applied to residues in TM3, TM6, TM9 and TM12 of each CFTR trajectory using snapshots taken every $1 \mathrm{~ns}$. A residue was classified as pore lining (i.e. solvent accessible) if its \%SASA was more than $20 \%{ }^{44}$

Pore analysis was conducted with the HOLE2 program $^{45}$ on snapshots taken every $0.5 \mathrm{~ns}$ using default parameters, except for the following: The "endrad" parameter was set to 10.0 and "cvect" was specified as the Z-axis (the direction of the pore). Each snapshot was manually inspected for the presence of a membrane traversing contiguous channel with the smallest radius $>0.2 \AA$ and non-zero HOLE2-predicted conductance (herein defined as open channel). Two types of channels were observed: (1) Channels spanning the entire length of the protein with a trajectory roughly perpendicular to the plane of the membrane, and (2) Channels following a perpendicular trajectory from the extracellular space until past the MSDs and following bending, a trajectory roughly parallel to the membrane with an exit point in the ICL region. This second type of channel is reminiscent of the bilateral channel reported by Mornon et al., ${ }^{23}$ Furthermore, each channel was required to neighbor at least three pore-lining residues as defined by Linsdell. ${ }^{46}$ The open probability of the different models was calculated as the number of snapshots with an open channel (as defined above) divided by the total number of the snapshots examined. 


\section{Results}

\subsection{MDFF simulations}

\subsubsection{General features of the refined models}

Three independent $20 \mathrm{~ns}$ MDFF simulations with different random seeds were initiated from the Dalton and Mornon models of human CFTR (a total of six simulations). The RMSD values with respect to the equilibrated structures as a function of the simulation progression are given in Figure 1 and demonstrate good convergence for most cases. (Note that the final jump in RMSD values at the last time point is due to the final minimization as described in section 2.2. Similar "jumps" in other calculated parameters are found in cross correlation (Figure 2) and salt bridges for the same reason). In almost all cases, the RMSD values between the initial and final models are between 5 and $6 \AA$ (note that in Figure 1, the starting value on the y-axis is $2.5 \AA$ indicating that the initial models changed their conformations already during the equilibration state). All simulations initiated from the same model converged to largely similar final models with averaged RMSD values of $3.1 \AA$ and $3.2 \AA$ for simulations initiated from the Dalton and Mornon models, respectively. The final models maintained good stereochemistry with $92.7 \% \pm 0.3 \%$ (Dalton) and $92.5 \% \pm 0.9 \%$ (Mornon) of their residues in the allowed regions of the Ramachandran plots. For comparison, the corresponding numbers for the initial Dalton and Mornon models and for the Sav1866 crystal structure are 97.8\%, 92.9\% and 92.1\%, respectively. The backbone RMSD between the two initial models was $5.6 \AA$ reflecting the differences between the homology modeling procedures used for their construction. Following MDFF simulations this number slightly changed to $5.7 \pm 0.4 \AA$ (based on a comparison between 1183 residue pairs) yet the two sets of models shared many common features.

During simulations, the models' cross correlation coefficient (CC) improved from 0.40 to $0.50 \pm$ 0.02 and from 0.42 to $0.53 \pm 0.01$ for the Dalton and Mornon models, respectively, reaching final values (following final minimization) of $0.57 \pm 0.01$ and $0.56 \pm 0.01$ (Figure 2). Chimera calculated cross correlation coefficients increased from 0.52 to $0.76 \pm 0.01$ and from 0.55 to 0.73 \pm 0.02 for the Dalton and Mornon models, respectively. Interestingly, fitting to the map has increased the asymmetry of the models. In particular in all simulations initiated from both models, helices 1 and 2 have slightly moved away from the pore to exploit a high electron density region of the map (circled in Figure 3). Thus the RMSD between the $\mathrm{N}$ - and C-termini 
halves increased from $11.7 \AA$ (initial model) to $12.6 \pm 0.2 \AA$ (final model) for the Dalton model and from $9.4 \AA$ (initial model) to $11.5 \pm 0.3 \AA$ (final model) for the Mornon model. This increase is in accord with the inherent asymmetry of the hetero-dimeric CFTR channel which is not present in the homo-dimeric Sav1866 template.

RMSD and cross correlation coefficient (CC) plots for the four control simulations (i.e., the simulations performed without the constraints imposed by the EM map) are provided in Figures S2 and S3, respectively. All graphs are indicative of good convergence. As expected for nonconstrained simulations, map fitting as judged by the cross correlation coefficient did not improve with time.

Consistent with the absence of ATP (and despite having their NBDs in a head-to-tail dimer conformation) the new models correspond to a closed channel conformation. In all MDFF simulations, channel closure resulted from an inward bending of helices 6 and 12, forming a tight hourglass conformation (Figure 4). The EM map and the newly derived models therefore suggest that a tight NBD1:NBD2 dimer is a viable CFTR conformation even in the absence of ATP which in turn implies that channel closure does not necessarily require the dissociation of the NBDs, at least not to a large degree. This proposition is in agreement with the "constant contact" model suggested for ABC transporters in general ${ }^{47}$ and also with the "limited NBD model" suggested specifically for CFTR. This model was based on the gating behavior of the CFTR channel. ${ }^{10}$ Notably, our results do not preclude a NBDs-separated conformation. Indeed, such a conformation was recently reported for zebrafish and human CFTR. ${ }^{15-16}$ Closure of the final model was not observed in the control simulations performed in the absence of ATP but without the map-derived constraints.

\subsubsection{Pore analysis}

Snapshots taken at $0.5 \mathrm{~ns}$ intervals from the resulting trajectories as well as the final models were analyzed for the existence of a contiguous (i.e., open) channel (see Methods section). In all channels considered to be open the radius of the pore was larger than $1.7 \AA$ (dehydrated chloride ion $)^{48}$ throughout most of the channel yet some channels were characterized by a small region with smaller pore radii. We reason that these regions may undergo structural changes e.g., upon 
the binding of ATP at the NBDs, resulting in a continuous pathway wide enough for ionic conduction. The combined results for both perpendicular and bent channels (see Methods section) are presented in Table 2 and suggest that the open probability of the CFTR channel during MDFF simulations is lower for both models than the open probabilities during the control simulations and the continuation simulations. (Of note, the term "open probability" is used here somewhat loosely. We do not claim that the MD simulations conducted in the work provide quantitative estimates of open probabilities. However, qualitative trends could be deduced.) The open probabilities of the channel in the control simulations (in the absence of ATP) are $0.29 \pm$ 0.06 and $0.40 \pm 0.05$ for Dalton and Mornon models, respectively, while the average open probabilities in the MDFF simulations are $0.18 \pm 0.10$ and $0.13 \pm 0.07$ for the Dalton and Mornon based simulations, respectively, with both final models not featuring a continuous channel. Interestingly, during the continuation simulations these values increased to $0.31 \pm 0.09$ and $0.33 \pm 0.07$ for Dalton and Mornon based simulations, respectively. Thus, the channel spends more time in a closed conformation in the MDFF simulations than in the control and in the continuation simulations. Taken together, these data suggest that channel closure results primarily from the constraints imposed by the EM map and not just from the absence of ATP.

\subsubsection{Agreement with experimentally determined salt bridges}

The final models as well as the resulting trajectories were examined for the existence of specific salt bridges which were experimentally observed in CFTR. The R347-D924 salt bridge is known to stabilize the structure of the pore and its removal leads to channel flickering between two conducting states. ${ }^{49}$ This salt bridge is found in the initial Dalton model and is maintained throughout all simulations initiated from it. Yet it is absent from one of the final models resulting from the final optimization due to a side-chain movement. This is an example of potential inconsistencies between results derived from MD trajectories and those obtained from single (perhaps arbitrary) snapshots stressing the need to analyze the complete trajectories. The R347D924 salt bridge is also found in the initial Mornon model (but not in the fully open model reported in that work), is maintained throughout all simulations initiated from it, but is absent from one of the final MDFF-derived models as well as from the final model obtained in the control simulation (Figure 5A\&B). 
The salt bridge between K1060 and E267 is responsible for holding together the tetrahelix bundle composed of ICLs 1-4. ${ }^{50}$ This salt bridge does not appear in the experimentally determined inward-facing conformation of CFTR due to the separation of the NBDs. This salt bridge exists in the initial Mornon model (and is borderline maintained in the "fully open" model, at $5.2 \AA$ separation), is maintained throughout most of the trajectories initiated from it, and is found in all final models. However, this salt bridge does not exist in the initial Dalton model yet it exists, at least partially, in all simulations initiated from this model as well as in two of the final models reflecting the NBD1:NBD2 close association. Thus, in this respect the MDFF-derived trajectory is in better agreement with the experimental data than was the initial model (Figure 5C\&D).

The R352-D993 salt bridge has a significant structural role in CFTR. Experimental data suggest that an interaction between these two residues is formed during channel opening and then broken during conformational changes underlying channel closure. ${ }^{51}$ This salt bridge is indeed found in the initial Dalton model but not in the initial Mornon model (however, it is found in the "fully open" model reported by Mornon as well as in the final models emerging from all control simulations reported in this work). In our simulations, channel closure primarily results from the coming together of helices 6 and 12 rather than from large helical movements or the dissociation of the NBDs. As a result, this salt bridge is largely maintained during the simulations as well as in most of the final models emerging from the two initial models. Nevertheless, in the simulations initiated from the Mornon model the distances between R352 and D993 are in order of 4-5 $\AA$, at the high end of what is considered for a salt bridge (Figure 5E\&F). ${ }^{52}$

\subsubsection{Agreement with experimentally determined cross linking data}

A comparison of cysteine cross linking data between experiment, the initial models (including the full open model reported by Mornon), the final models, and the trajectories is provided in Table S1. Overall, there are no significant changes following MDFF simulations except for a decrease in the distance between TM6 and TM12, in accord with channel closure (Figure 4). Yet even these shorter values are still in agreement with experiment. 
Recently, Negoda et al., have used cysteine cross-linking to study the so-called selectivity filter of CFTR suggesting distances smaller than $7 \AA$ between the C $\alpha$ carbons of L102 (TM1) and F337 (TM6) and between the C $\alpha$ carbons of L102 and F338 (TM6). ${ }^{53}$ Since patch clamp recordings were used to identify changes in channel conductance resulting from the formation of the cys-cys bonds, these distances likely correspond to CFTR in its open (i.e., conducting) state. Yet the corresponding distances found in the models used as starting points for the MDFF simulations (as well as in the fully open state reported by Mornon) are considerably longer (L102-F337 = 19.7 $\AA, 18.0 \AA$, and $15.7 \AA$ for the Mornon-open, Mornon-full open and Dalton models, respectively and L102-F338 = $18.8 \AA, 17.5 \AA$, and $14.4 \AA$ for the Mornon-open, Mornon-fully open and Dalton models, respectively). Analyzing whether this discrepancy results from inaccuracies in the initial models or from the experimental setup (as noted in the paper, some discrepancies exit between the cysteine cross linking results and metal bridge results) is beyond the scope of the present work. The large distances observed in the initial models were carried over to the final models (L102-F337 $=20.5 \pm 1.5 \AA$ and $23.2 \pm 2.6 \AA$ for the Mornon and Dalton models, respectively and L102-F338 $=18.3 \pm 1.0 \AA$ and $20.9 \pm 2.4 \AA$ for the Mornon and Dalton models, respectively). As discussed in this work, these models likely correspond to a closed-state CFTR conformation whereas Negoda et al. conducted their studies on open-state CFTR (see above). Thus no firm conclusions could be drawn regarding the correspondence between the models and the experimental data. Interestingly, much shorter distances are observed in human CFTR EM structure (L102-F337 = $8.9 \AA$; L102-F338 = $7.0 \AA$ ) and in the closed CFTR structure reported by Mornon $(\mathrm{L} 102-\mathrm{F} 337=9.8 \AA \AA$; L102-F338 = 8.6 ̊̊). Figure S4 provides a comparison of the proposed narrow-pore lining residues between the EM structure, the initial models, the final models and the closed state CFTR model reported by Mornon.

\subsubsection{Agreement with experimentally determined hydrogen bond}

Residues R555 and T1246 at the NBD1:NBD2 dimer interface are known to be coupled in the open channel state via hydrogen bond interaction. ${ }^{54}$ Accordingly, this hydrogen bond exists in the initial Dalton model, yet in only $\sim 50 \%$ of the simulations initiated from it. This hydrogen bond is also found in the final models, stressing once more the need to rely on trajectories rather than on individual structures. We propose that the partial occurrence of this hydrogen bond in the MDFF simulations is due to channel closure resulting from movement of the helices rather than 
NBDs. This hydrogen bond is absent from the initial Mornon model and appears at very low frequencies only in the continuation simulations.

\subsubsection{Agreement with experimentally determined solvent accessible surface area (SASA) data} Each residue from TM3, TM6, TM9 and TM12 was analyzed for its solvent accessible surface area (SASA) based on snapshots taken from the MDFF and MD trajectories and the results were compared with the experimental data as reported by Norimatsu et al. ${ }^{44}$ (Table 3A-D). Notably, this comparison is largely indirect since most data on probe accessibility were accumulated in the presence of ATP and consequently correspond to the open state of the channel. Yet several residues on TM12 are known to be exposed to a more hydrophobic environment in closed channels, namely residues $1141,1142,1145,1147$ and $1150 .{ }^{55}$ In accord with this observation, residues 1141, 1145 and 1150 have lower predicted averaged \%SASA values in the MDFF simulations than in both initial models. Residue 1142 has a lower predicted \%SASA only in the MDFF simulations initiated from the Dalton model, whereas residue 1147 has higher \%SASA in MDFF simulations initiated from both models, but still lower than the cutoff value of $20 \%$.

As noted above, the rest of the experimental data presented in Tables 3A-D were obtained in the presence of ATP. We therefore expect that the percentage of agreement of the predicted \%SASA with experiment will be lower for the MDFF simulations, which correspond to the closed state, than for the open state initial models. In accord with this proposition, the agreement of the predicted \%SASA with experiment is indeed lower for snapshots taken from the MDFF trajectory in all cases except for TM3 (Mornon) and TM6 (Dalton).

\section{Discussion and Conclusions}

In this work we present for the first time the application of MDFF simulations to the refinement of two of the outward facing published CFTR models based on cryo electron microscopy data recorded for the human protein. We chose to focus on the EMD-1966 map since it corresponds to a CFTR conformation which to date was largely unexplored yet one which is likely relevant to the gating cycle of the channel (see below). This map is distinctively different from the zebrafish and human CFTR maps reported by the Chen $\mathrm{lab}^{15}$ and consequently the resulting models are different from the published structures. The decision to refine the reported pre-MD rather than 
the post-MD structures was based on two considerations. First, our intention was not to refine the "best" open CFTR model but rather a model having the NBDs closely associated, since this was the conformation of the protein suggested by the EM map. Furthermore, since the map was recorded in the absence of ATP it likely corresponds to a closed state CFTR. Thus we saw no reason to refine a model that was "optimized" for an open state either through unbiased MD simulation (i.e., Monon model) or by the (non-physical) introduction of a file of chloride ions (i.e., Dalton model). Second, the Dalton paper only provided the coordinates of the pre-MD model. For the sake of consistency we therefore decided to use the corresponding model from the Mornon paper as well.

To obtain good modeling statistics, each MDFF simulation was repeated three times for a total of $60 \mathrm{~ns}$ for each starting model. We deem these simulations to be of sufficient length in particular due to the map-imposed constraints which prevent large excursions from the boundaries of the map. In agreement with this proposition, good convergence was obtained in all cases and all simulations initiated from a specific model converged to similar final structures (averaged RMSD between final models is $3.1 \AA$ and $3.2 \AA$ for simulations initiated from the Dalton and Mornon models, respectively). The final models as well as the trajectories are in reasonably good agreement with the available experimental data. While the final models are not overwhelmingly different from their respective starting points in terms of RMSD values (5-6 $\AA$ ), the differences are large enough to reflect significant conformational changes, i.e., an open channel to closed channel transition. Small structural differences which lead to large functional effects are not uncommon in the CFTR field. As an example, the RMSD between the crystal structures of wtNBD1 and F508del-NBD1 is a mere $0.6 \AA$. Yet these seemingly similar structures behave in very different ways in terms e.g., of their thermal stabilities and aggregation propensities. ${ }^{56-57}$

The importance of this work is two-fold. First it provides new conformations of CFTR at the atomic level which were largely not discussed to date. These conformations were modeled under conditions similar to those used for the recording of the EM map (i.e., absence of ATP) and feature an outward facing arrangement of the TMs with a tight NBD1:NBD2 dimer yet with a closed channel. These conformations are therefore distinctly different from the closed state 
structures recently reported for zebrafish and human CFTR. These conformations are reminiscent of the closed CFTR structure recently reported by Mornon et al., yet their overall structures are different (RMSD between the closed conformation reported by Mornon and the Mornon-based and Dalton-based models reported in this work is $7.6 \pm 0.4 \AA$ and $9.2 \pm 0.1 \AA$, respectively). Specifically, in the CFTR conformations reported in this work, channel closure results from the coming together of helices 6 and 12 rather than from the movement of the side chain of F337 as reported by Mornon et al. Additional differences between the Mornon closed model and the models reported in this work are found in the NBDs. In particular residues in the vicinity of the ATP sites moved to fill the void formed by the removal of ATP. Of note is that all ATP-free simulations (i.e., those conducted with and without the EM constraints) demonstrated similar distortions in the vicinity of the ATP site suggesting the resulting structures to be faithful models of ATP-less CFTR. The backbone RMSD between the ATP-less models and the initial models calculated for residues within $5 \AA$ of both ATP sites is $3.9 \pm 0.5 \AA$ and $4.1 \pm 0.2 \AA$ for the Mornon and Dalton models, respectively (based on a comparison of 52 residue pairs), suggesting that rather small structural changes are required to make the new models ATP-compatible. Finally, the new conformations feature a higher degree of asymmetry between their N-terminus and C-terminus halves in comparison with the initial structures reflecting the hetero-dimeric nature of human CFTR. In accord with this proposition, the RMSD difference between the two halves of the hetero-dimeric TM287-288 ABC transporter structure is $18 \AA$. The use of MDFF simulations may, therefore, correct for the fact that the models of full-length CFTR (containing two MSDs and two NBDs) were generated based on a homodimer of full-length Sav1866 (which contains only one MSD and one NBD per monomer).

The potential physiological relevance of these new conformations merits some discussion. The CFTR gating cycle is controlled by R-region phosphorylation / de-phosphorylation and by ATP binding to and hydrolysis from the canonical and non-canonical sites at the NBD1:NBD2 interface (with residence time in the non-canonical site being considerably longer than in the canonical site). ${ }^{10,58}$ The CFTR conformations presented in this work are compatible with a model whereby priming of CFTR by phosphorylation is linked to large conformational changes (i.e., from inward facing to outward facing), while rapid channel opening and closing is associated with small allosteric movements controlled by ATP binding and dissociation events 
which do not require NBD1:NBD2 dimer dissociation. This model is compatible with the "limited NBD model" suggested for CFTR based on the gating behavior of the channel ${ }^{10}$ as well as with the Mornon model suggesting chloride diffusion into the CFTR conducting pore via a lateral tunnel. ${ }^{21}$ In addition, while for most $\mathrm{ABC}$ transporters that translocate large substances across the cell membrane an NBDs-separated conformation might be a necessity to allow substance diffusion into the "transporting chamber", 59 this is not necessarily the case for CFTR where the small size of the transported substance (i.e., chloride or bicarbonate ions) may not require large separation of the NBDs. Finally, a model in which the NBDs are closely associated even in the closed state raises questions regarding the entry pathway of ATP and the exit pathway of ADP. Answering such questions at the computational level will likely require lengthy MD simulations. Such simulations are currently being conducted in our laboratory.

Second, the ability to use EM maps for model refinement is important for several reasons: (1) EM maps constitute a source of experimental data different from what has been used to date for model construction and validation. (2) EM maps could be "transferred" into relevant models at atomic resolutions. In this respect, medium to high resolution EM maps may have an advantage over a single snapshot obtained, e.g., from X-ray crystallography or cryo-EM, since such maps could be regarded as an envelope within which the protein can move. Such movements (i.e., protein flexibility) are key to CFTR function. MDFF simulations generate trajectories that could capture these movements. For this reason, in the present study we analyzed complete MDFF trajectories rather than focusing on end structures only. We realize however that for the purpose of structure-based drug discovery, one should rely on a single or on a limited set of structures. (3) While the human wt-CFTR EM map used in this study is only of low resolution, additional maps with much better resolutions for different CFTR constructs are quickly becoming available. ${ }^{15-16,32}$ Efforts towards the development of such maps take advantage of impressive improvements in EM technology and the concomitant recording of medium to high resolution EM maps of CFTR and its homologues. ${ }^{15-16,60-61}$ Moreover, recording maps in the presence and absence of known CFTR modulators could inform on their binding sites. A combination of MDFF and docking simulations could therefore be used to elucidate the binding modes of these modulators and to suggest ways to improve them, paving the way to structure-based drug design. MDFF simulations could also be performed under additional constraints derived from other 
experimental sources to produce CFTR models with yet improved fit to experiment. In particular, data pertaining to the R-region and the C-terminus could help in fitting these largely disordered domains into high density map regions not accounted for by other CFTR domains. ${ }^{62-63}$ Indeed such regions are clearly visible in the zebrafish CFTR map and a small part of the Rregion was modeled into the EM map of human CFTR (PDB code 5UAK). Finally, a variant of MDFF simulations, xMDFF, was recently introduced which would allow for structure refinement from low-resolution x-ray crystallographic diffraction data, once these become available for human CFTR. ${ }^{64}$

Several limitations of MDFF simulations should be noted: (1) clearly the resolution of a model refinement based on the map cannot be higher than the resolution of the map (unless additional constraints derived from other experimental data are added). However, based on the above discussion we do not necessarily consider a limited "vagueness" to be a drawback. (2) MDFF simulations are inherently biased by the usage of a specific homology model. Yet, in the case of CFTR where multiple experimental data support the overall fold of current homology models, we do not see this as a major source of concern. In agreement with this proposition, the recently solved structures of zebrafish and human CFTR have a fold generally similar to other inward facing $\mathrm{ABC}$ transporters. (3) MDFF simulations rely on EM densities which should be free from non-physiological biases and obtained under physiologically relevant conditions. We have discussed in the Introduction section the potential biases of the current map and the way the map was processed in order to mitigate them. Inasmuch as the map is relevant, MDFF simulations can take advantage of its features.

Finally, we wish to touch upon the topic of model validation which is likely to remain relevant at least until high resolution structures of CFTR in multiple conformations become available. A wealth of experimental information is currently available for CFTR including (non-exhaustively) electron microscopy data, NMR data, mutational data, cysteine cross linking data, hydrogen bonding and salt bridge data, probe accessibility data, and data pertaining to the binding of open channel blockers. Yet these data are: (1) scattered around multiple publications and, (2) were obtained under different conditions and consequently likely pertain to different states of CFTR. 
Many of these data were used to validate CFTR models yet in a non-consistent way with different models being evaluated against different data. Thus we advocate the compilation of a, state-dependent set of experimental data to validate CFTR models. Work along these lines in currently being conducted in our laboratory and others.

In sum, in this work we have demonstrated the usefulness of MDFF simulations in identifying new CFTR conformations with good stereochemical parameters, favorable agreement with experimental data and potential physiological relevance. These models fit the EM-derived densities significantly better than previously reported models. We propose that MDFF simulations will find value in refining models based on EM maps with higher resolutions. We further propose that the combination of electron microscopy, MDFF simulations and docking studies could identify ligand binding sites on CFTR models thereby advancing structure-based drug discovery in the CF arena.

Supporting Information Available: Figure S1-S4 and Table S1. Also available are the six final models (three for the Mornon model (Mornon_final_1-3) and three for the Dalton model (Dalton_final_1-3) and 10 equally spaced snapshots from each trajectory (Mornon_trj_1-3, Dalton_trj_1-3) from the MDFF simulations (PDB format). This material is available free of charge via the Internet at http://pubs.acs.org.

\section{Acknowledgment}

We wish to thank our colleagues at the CFF CFTR 3D structure consortium for many helpful discussions. This work was supported by the Cystic Fibrosis Foundation Therapeutics, grant No. SENDER13XX0 to H. Senderowitz, and by the National Institutes of Health, grants NIH R01DK 056481 and R01-DK 075016 to N. McCarty 


\section{References}

1. Cheung, J. C.; Deber, C. M. Misfolding of the Cystic Fibrosis Transmembrane Conductance Regulator and Disease. Biochemistry 2008, 47, 1465-1473.

2. Lubamba, B.; Dhooghe, B.; Noel, S.; Leal, T. Cystic Fibrosis: Insight into CFTR Pathophysiology and Pharmacotherapy. Clin. Biochem. 2012, 45, 1132-1144.

3. Castellani, C. CFTR2: How Will It Help Care? Paediatr. Respir. Rev. 2013, 14, , 2-5.

4. Frizzell, R. A.; Hanrahan, J. W. Physiology of Epithelial Chloride and Fluid Secretion. Cold Spring Harbor Perspect. Med. 2012, 2, a009563.

5. Bompadre, S. G.; Hwang, T. C. Cystic Fibrosis Transmembrane Conductance Regulator: A Chloride Channel Gated by ATP Binding and Hydrolysis. Acta Physiol. Sin. 2007, 59, 431-442.

6. Rees, D. C.; Johnson, E.; Lewinson, O. ABC Transporters: The Power to Change. Nat. Rev.. Mol. Cell Biol. 2009, 10), 218-227.

7. McCarty, N. A. Permeation Through the CFTR Chloride Channel. J. Exp.I Biol. 2000, 203, 19471962.

8. Liu, X.; Smith, S. S.; Dawson, D. C. CFTR: What's It Like Inside the Pore? J. Exp. Zool, Part A, 2003, $300,69-75$.

9. Linsdell, P. Mechanism of Chloride Permeation in the Cystic Fibrosis Transmembrane Conductance Regulator Chloride Channel. Exp. Physiol. 2006, 91, 123-129.

10. Hwang, T.-C.; Kirk, K. L. The CFTR Ion Channel: Gating, Regulation, and Anion Permeation. Cold Spring Harbor Perspect. Med. 2013, 3, a009498.

11. Kos, V.; Ford, R. C. The ATP-Binding Cassette Family: A Structural Perspective. Cell. Mol. Life Sci. 2009, 66, 3111-3126.

12. Locher, K. P. Structure and Mechanism of ATP-Binding Cassette Transporters. Philos. Trans. $R$. Soc., B 2009, 364, 239-245.

13. Rowe, S. M.; Verkman, A. S. Cystic Fibrosis Transmembrane Regulator Correctors and Potentiators. Cold Spring Harbor Perspect. Med. 2013, 3.

14. Rahman, K. S.; Cui, G.; Harvey, S. C.; McCarty, N. A. Modeling the Conformational Changes Underlying Channel Opening in CFTR. PLOS ONE 2013, 8, e74574.

15. Zhang, Z.; Chen, J. Atomic Structure of the Cystic Fibrosis Transmembrane Conductance Regulator. Cell 2016, 167, 1586-1597.e9.

16. Liu, F.; Zhang, Z.; Csanády, L.; Gadsby, D. C.; Chen, J. Molecular Structure of the Human CFTR Ion Channel. Cell 169, 85-95.e8.

17. Mornon, J. P.; Lehn, P.; Callebaut, I. Atomic Model of Human Cystic Fibrosis Transmembrane Conductance Regulator: Membrane-spanning Domains and Coupling Interfaces. Cell. Mol. Life Sci. 2008, 65, 2594-2612.

18. Serohijos, A. W.; Hegedus, T.; Aleksandrov, A. A.; He, L.; Cui, L.; Dokholyan, N. V.; Riordan, J. R. Phenylalanine-508 Mediates a Cytoplasmic-membrane Domain Contact in the CFTR 3D Structure Crucial to Assembly and Channel Function. Proc. Natl. Acad. Sci. U. S. A. 2008, 105, 3256-3261.

19. Mornon, J. P.; Lehn, P.; Callebaut, I. Molecular Models of the Open and Closed States of the Whole Human CFTR Protein. Cell. Mol. Life Sci. 2009, 66, 3469-3486.

20. Dalton, J.; Kalid, O.; Schushan, M.; Ben-Tal, N.; Villa-Freixa, J. New Model of Cystic Fibrosis Transmembrane Conductance Regulator Proposes Active Channel-Like Conformation. J. Chem. Inf. Model. 2012, 52, 1842-1853.

21. Norimatsu, Y.; Ivetac, A.; Alexander, C.; Kirkham, J.; O'Donnell, N.; Dawson, D. C.; Sansom, M. S. Cystic Fibrosis Transmembrane Conductance Regulator: A Molecular Model Defines the Architecture of the Anion Conduction Path and Locates a "Bottleneck" in the Pore. Biochemistry 2012, 51, 2199-2212. 
22. Furukawa-Hagiya, T.; Furuta, T.; Chiba, S.; Sohma, Y.; Sakurai, M. The Power Stroke Driven by ATP Binding in CFTR As Studied by Molecular Dynamics Simulations. J. $P$ hys. Chem. B 2013, 117, 83-93.

23. Mornon, J.-P.; Hoffmann, B.; Jonic, S.; Lehn, P.; Callebaut, I. Full-open and Closed CFTR Channels, With Lateral Tunnels From the Cytoplasm and an Alternative Position of the F508 Region, As Revealed by Molecular Dynamics. Cell. Mol. Life Sci. 2015, 72, 1377-1403.

24. Dawson, R. J.; Locher, K. P. Structure of a Bacterial Multidrug ABC Transporter. Nature 2006, $443,180-185$.

25. Choudhury, H. G.; Tong, Z.; Mathavan, I.; Li, Y.; Iwata, S.; Zirah, S.; Rebuffat, S.; van Veen, H. W.; Beis, K. Structure of an Antibacterial Peptide ATP-Binding Cassette Transporter in a Novel Outward Occluded State. Proc. Natl. Acad. Sci. U. S. A. 2014, 111, 9145-9150.

26. Corradi, V.; Vergani, P.; Tieleman, D. P. Cystic Fibrosis Transmembrane Conductance Regulator (CFTR): Closed and Open State Channel Models. J. Biol. Chem 2015, 290, 22891-22906.

27. Ward, A.; Reyes, C. L.; Yu, J.; Roth, C. B.; Chang, G. Flexibility in the ABC Transporter MsbA: Alternating Access with a Twist. Proc. Natl. Acad. Sci.U. S. A. 2007, 104, 19005-19010.

28. Aller, S. G.; Yu, J.; Ward, A.; Weng, Y.; Chittaboina, S.; Zhuo, R.; Harrell, P. M.; Trinh, Y. T.; Zhang, Q.; Urbatsch, I. L.; Chang, G. Structure of P-glycoprotein Reveals a Molecular Basis for Poly-Specific Drug Binding. Science 2009, 323, 1718-1722.

29. Hohl, M.; Hürlimann, L. M.; Böhm, S.; Schöppe, J.; Grütter, M. G.; Bordignon, E.; Seeger, M. A. Structural Basis for Allosteric Cross-talk Between the Asymmetric Nucleotide Binding Sites of a Heterodimeric ABC Exporter. Proc. Natl.IAcad.Sci. U. S. A. 2014, 111, 11025-11030.

30. Shintre, C. A.; Pike, A. C. W.; Li, Q.; Kim, J.-I.; Barr, A. J.; Goubin, S.; Shrestha, L.; Yang, J.; Berridge, G.; Ross, J.; Stansfeld, P. J.; Sansom, M. S. P.; Edwards, A. M.; Bountra, C.; Marsden, B. D.; von Delft, F.; Bullock, A. N.; Gileadi, O.; Burgess-Brown, N. A.; Carpenter, E. P. Structures of ABCB10, A Human ATP-Binding Cassette Transporter in Apo- and Nucleotide-bound States. Proc. Natl. Acad. Sci. U. S.A. 2013, 110, 9710-9715.

31. Rosenberg, M. F.; O'Ryan, L. P.; Hughes, G.; Zhao, Z.; Aleksandrov, L. A.; Riordan, J. R.; Ford, R. C. The Cystic Fibrosis Transmembrane Conductance Regulator (CFTR): Three-dimensional Structure and Localization of a Channel Gate. J. Biol. Chem 2011, 286, 42647-42654.

32. Ford, B. C., R.F.; Meng, X. , G551D CFTR 3D Structure Determined by Cryo-Electron Microscopy. Pediatr. Pulmonol. 2016, 51, S195.

33. Ford, R. C.; Holzenburg, A. Electron Crystallography of Biomolecules: Mysterious Membranes and Missing Cones. Trends Biochem. Sci. 2008, 33, 38-43.

34. Trabuco, L. G.; Villa, E.; Schreiner, E.; Harrison, C. B.; Schulten, K. Molecular Dynamics Flexible Fitting: A Practical Guide to Combine Cryo-electron Microscopy and X-ray Crystallography. Methods 2009, 49, 174-180.

35. Inc., A. S., Discovery Studio 3.5. San Diego: Accelrys Software Inc. 2010.

36. Lomize, M. A.; Pogozheva, I. D.; Joo, H.; Mosberg, H. I.; Lomize, A. L. OPM Database and PPM Web Server: Resources for Positioning of Proteins in Membranes. Nucleic Acids Res. 2012, 40, D370-376.

37. Jorgensen, W. L.; Chandrasekhar, J.; Madura, J. D.; Impey, R. W.; Klein, M. L. Comparison of Simple Potential Functions for Simulating Liquid Water. J. Chem. Phys. 1983, 79, 926-935.

38. Humphrey, W.; Dalke, A.; Schulten, K. VMD: Visual Molecular Dynamics. J. Mol. Graphics 1996, 14, 33-38.

39. Phillips, J. C.; Braun, R.; Wang, W.; Gumbart, J.; Tajkhorshid, E.; Villa, E.; Chipot, C.; Skeel, R. D.; Kale, L.; Schulten, K. Scalable Molecular Dynamics with NAMD. J. Comput. Chem. 2005, 26, 1781-1802.

40. Huang, J.; MacKerell, A. D., Jr. CHARMM36 All-atom Additive Protein Force Field: Validation Based on Comparison to NMR Data. J. Comput. Chem. 2013, 34, 2135-2145. 
41. Darden, T.; York, D.; Pedersen, L. Particle Mesh Ewald: An N.log(N) Method for Ewald Sums in Large Systems. J. Chem. Phys. 1993, 98, 10089-10092.

42. Wriggers, W. Conventions and Workflows for Using Situs. Acta Crystallogr., Sect. D: Biol. Crystallogr. 2012, 68, 344-351.

43. Pettersen, E. F.; Goddard, T. D.; Huang, C. C.; Couch, G. S.; Greenblatt, D. M.; Meng, E. C.; Ferrin, T. E. UCSF Chimera:A Visualization System for Exploratory Research and Analysis. J. Comput. Chem. 2004, 25, 1605-1612.

44. Alexander, C.; Ivetac, A.; Liu, X.; Norimatsu, Y.; Serrano, J. R.; Landstrom, A.; Sansom, M.; Dawson, D. C. Cystic Fibrosis Transmembrane Conductance Regulator: Using Differential Reactivity Toward Channel-Permeant and Channel-Impermeant Thiol-Reactive Probes to Test a Molecular Model for the Pore. Biochemistry 2009, 48, 10078-10088.

45. Smart, O. S.; Goodfellow, J. M.; Wallace, B. A. The Pore Dimensions of Gramicidin A. Biophys. J. 1993, 65, 2455-2460.

46. Linsdell, P. Architecture and Functional Properties of the CFTR Channel Pore. Cell. Mol. Life Sci. 2016, 1-17.

47. George, A. M.; Jones, P. M. Perspectives on the Structure-Function of ABC Transporters: The Switch and Constant Contact Models. Prog. Biophys. Mol. Biol. 2012, 109, 95-107.

48. Marcus, Y., Ion properties. Marcel Dekker: New York, 1997.

49. Cotten, J. F.; Welsh, M. J. Cystic Fibrosis-associated Mutations at Arginine 347 Alter the Pore Architecture of CFTR: Evidence for Disruption of a Salt Bridge. J. Biol. Chem. 1999, 274, 5429-5435.

50. Wang, W.; Roessler, B. C.; Kirk, K. L. An Electrostatic Interaction at the Tetrahelix Bundle Promotes Phosphorylation-dependent Cystic Fibrosis Transmembrane Conductance Regulator (CFTR) Channel Opening. J. Biol. Chem. 2014, 289, 30364-30378.

51. Cui, G.; Zhang, Z. R.; O'Brien, A. R.; Song, B.; McCarty, N. A. Mutations at Arginine 352 Alter the Pore Architecture of CFTR. J. Membr. Biol. 2008, 222, 91-106.

52. Kumar, S.; Nussinov, R. Relationship Between Ion Pair Geometries and Electrostatic Strengths in Proteins. Biophys. J. 2002, 83, 1595-1612.

53. Negoda, A.; El Hiani, Y.; Cowley, E. A.; Linsdell, P. Contribution of a Leucine Residue in the First Transmembrane Segment to the Selectivity Filter Region in the CFTR Chloride Channel. Biochim. Biophys. Acta, Biomembr. 2017, 1859, 1049-1058.

54. Vergani, P.; Lockless, S. W.; Nairn, A. C.; Gadsby, D. C. CFTR Channel Opening by ATP-driven Tight Dimerization of Its Nucleotide-binding Domains. Nature 2005, 433, 876-880.

55. Bai, Y.; Li, M.; Hwang, T.-C. Structural Basis for the Channel Function of a Degraded ABC Transporter, CFTR (ABCC7). J. Gen Physiol. 2011, 138, 495-507.

56. Wang, C.; Protasevich, I.; Yang, Z.; Seehausen, D.; Skalak, T.; Zhao, X.; Atwell, S.; Spencer Emtage, J.; Wetmore, D. R.; Brouillette, C. G.; Hunt, J. F. Integrated Biophysical Studies Implicate Partial Unfolding of NBD1 of CFTR in the Molecular Pathogenesis of F508del Cystic Fibrosis. Protein Sci. 2010, 19, 1932-1947.

57. Protasevich, I.; Yang, Z.; Wang, C.; Atwell, S.; Zhao, X.; Emtage, S.; Wetmore, D.; Hunt, J. F.; Brouillette, C. G. Thermal Unfolding Studies Show the Disease Causing F508del Mutation in CFTR Thermodynamically Destabilizes Nucleotide-Binding Domain 1. Protein Sci. 2010, 19, 1917-1931.

58. Aleksandrov, L.; Aleksandrov, A. A.; Chang, X. B.; Riordan, J. R. The First Nucleotide Binding Domain of Cystic Fibrosis Transmembrane Conductance Regulator Is a Site of Stable Nucleotide Interaction, whereas the Second Is a Site of Rapid Turnover. J. Biol. Chem. 2002, 277, 15419-15425.

59. Hollenstein, K.; Dawson, R. J. P.; Locher, K. P. Structure and Mechanism of ABC Transporter Proteins. Curr. Opin. Struct. Biol. 2007, 17, 412-418. 
60. Kim, J.; Wu, S.; Tomasiak, T.; Mergel, C.; Winter, M. B.; Stiller, S. B.; Robles-Colmanares, Y.; Stroud, R. M.; Tampé, R.; Craik, C. S.; Cheng, Y. Subnanometer Resolution Cryo-EM Structure of a Nucleotide Free Heterodimeric ABC Exporter. Nature 2015, 517, 396-400.

61. Oldham, M. L.; Hite, R. K.; Steffen, A. M.; Damko, E.; Li, Z.; Walz, T.; Chen, J. A Mechanism of Viral Immune Evasion Revealed by Cryo-EM Analysis of the TAP Transporter. Nature 2016, 529, 537-540.

62. Kanelis, V.; Chong, P. A.; Forman-Kay, J. D. NMR Spectroscopy to Study the Dynamics and Interactions of CFTR. In Cystic Fibrosis: Diagnosis and Protocols, Volume I: Approaches to Study and Correct CFTR Defects, Amaral, D. M.; Kunzelmann, K., Eds. Humana Press: Totowa, NJ, 2011; pp 377-403. 63. Bozoky, Z.; Krzeminski, M.; Muhandiram, R.; Birtley, J. R.; Al-Zahrani, A.; Thomas, P. J.; Frizzell, R. A.; Ford, R. C.; Forman-Kay, J. D. Regulatory R region of the CFTR Chloride Channel Is a Dynamic Integrator of Phospho-dependent Intra- and Intermolecular Interactions. Proc. Natl. Acad. Sci. U. S. A. 2013, 110, E4427-E4436.

64. McGreevy, R.; Singharoy, A.; Li, Q.; Zhang, J.; Xu, D.; Perozo, E.; Schulten, K. xMDFF: Molecular Dynamics Flexible Fitting of Low-resolution X-ray Structures. Acta Crystallogr., Sect. D: Biol. Crystallogr. 2014, 70, 2344-2355. 
Table 1. Backbone RMSD $(\AA)$ differences between reported CFTR models. Models [1, 2, 3 and 12], [4-6] and [13-14], [7-9], [10-11] and [15-16] were taken from reference 17, 19, 18, 14, 22, 25 , respectively.

\begin{tabular}{|c|c|c|c|c|c|c|c|c|c|c|c|c|c|c|c|c|}
\hline & \multicolumn{11}{|c|}{ OUTWARD FACING } & \multicolumn{5}{|c|}{ INWARD FACING } \\
\hline & 1 & 2 & 3 & 4 & 5 & 6 & 7 & 8 & 9 & 10 & 11 & 12 & 13 & 14 & 15 & 16 \\
\hline 1 & & 6.4 & 22.8 & 3.8 & 4.0 & 4.7 & 6.1 & 7.6 & 7.7 & 4.4 & 5.8 & & & & & \\
\hline 2 & 6.4 & & 4.4 & 6.0 & 5.9 & 6.3 & 5.7 & 7.0 & 8.1 & 3.3 & 5.0 & & & & & \\
\hline 3 & 22.8 & 4.4 & & 5.5 & 5.6 & 6.1 & 5.5 & 6.7 & 8.4 & 3.0 & 5.3 & & & & & \\
\hline 4 & 3.8 & 6.0 & 5.5 & & 1.5 & 3.4 & 5.4 & 7.3 & 8.2 & 4.0 & 6.7 & & & & & \\
\hline 5 & 4.0 & 5.9 & 5.6 & 1.5 & & 2.9 & 5.4 & 7.2 & 8.1 & 4.0 & 6.7 & & & & & \\
\hline 6 & 4.7 & 6.3 & 6.1 & 3.4 & 2.9 & & 6.0 & 7.3 & 8.2 & 4.8 & 7.0 & & & & & \\
\hline 7 & 6.1 & 5.7 & 5.5 & 5.4 & 5.4 & 6.0 & & 5.0 & 6.4 & 3.1 & 5.8 & & & & & \\
\hline 8 & 7.6 & 7.0 & 6.7 & 7.3 & 7.2 & 7.3 & 5.0 & & 5.6 & 5.5 & 6.2 & & & & & \\
\hline 9 & 7.7 & 8.1 & 8.4 & 8.2 & 8.1 & 8.2 & 6.4 & 5.6 & & 6.3 & 6.2 & & & & & \\
\hline 10 & 4.4 & 3.3 & 3.0 & 4.0 & 4.0 & 4.8 & 3.1 & 5.5 & 6.3 & & 5.6 & & & & & \\
\hline 11 & 5.8 & 5.0 & 5.3 & 6.7 & 6.7 & 7.0 & 5.8 & 6.2 & 6.2 & 5.6 & & & & & & \\
\hline 12 & & & & & & & & & & & & & 11.7 & 10.0 & 9.4 & 6.7 \\
\hline 13 & & & & & & & & & & & & 11.7 & & 5.3 & 6.5 & 7.9 \\
\hline 14 & & & & & & & & & & & & 10.0 & 5.3 & & 5.7 & 6.5 \\
\hline 15 & & & & & & & & & & & & 9.4 & 6.5 & 5.7 & & 4.5 \\
\hline 16 & & & & & & & & & & & & 6.7 & 7.9 & 6.5 & 4.5 & \\
\hline
\end{tabular}

Table 2. Open probabilities of the CFTR channel (perpendicular and bent) during simulations. See methods section for more details.

\begin{tabular}{l|c|c} 
& \multicolumn{2}{|c}{ OPEN CHANNEL PROBABILITY } \\
\hline SIMULATION & Dalton-Based & Mornon-Based \\
\hline CONTROL WITHOUT ATP & $0.29 \pm 0.06$ & $0.40 \pm 0.05$ \\
MDFF & $0.18 \pm 0.10$ & $0.13 \pm 0.07$ \\
CONTINUATION MD & $0.31 \pm 0.09$ & $0.33 \pm 0.07$
\end{tabular}


Table 3. Comparison of percent of solvent accessible surface area (\%SASA) of amino acids from TM3 (A), TM6 (B), TM9 (C) and TM12 (D) deduced from experiment, the initial models, MDFF/MD simulations, and the final models. (•): Amino acids are experimentally observed to be solvent accessible; (-): Amino acids are experimentally observed not to be solvent accessible. A residue was classified as solvent accessible if its \%SASA is more than $20 \%$.

A.

\begin{tabular}{|c|c|c|c|c|c|c|c|c|c|c|c|}
\hline \multirow[b]{2}{*}{ RESIDUE } & \multirow[b]{2}{*}{ EXP } & \multicolumn{5}{|c|}{ DALTON } & \multicolumn{5}{|c|}{ MORNON } \\
\hline & & $\begin{array}{l}\text { Initial } \\
\text { Model }\end{array}$ & $\begin{array}{c}\text { Control } \\
\text { MD }\end{array}$ & MDFF & $\begin{array}{c}\text { Final } \\
\text { Model }\end{array}$ & $\begin{array}{c}\text { Continuation } \\
\text { MD }\end{array}$ & $\begin{array}{l}\text { Initial } \\
\text { Model }\end{array}$ & $\begin{array}{c}\text { Control } \\
\text { MD }\end{array}$ & MDFF & $\begin{array}{c}\text { Final } \\
\text { Model }\end{array}$ & $\begin{array}{c}\text { Continuation } \\
\text { MD }\end{array}$ \\
\hline LYS190 & $\bullet$ & 69 & $52 \pm 12$ & $22 \pm 4$ & $18 \pm 4$ & $36 \pm 9$ & 26 & $37 \pm 6$ & $26 \pm 5$ & $19 \pm 3$ & $38 \pm 8$ \\
\hline PHE191 & $\bullet$ & 4 & $7 \pm 3$ & $4 \pm 2$ & $8 \pm 4$ & $4 \pm 3$ & 3 & $13 \pm 4$ & $19 \pm 4$ & $24 \pm 9$ & $12 \pm 6$ \\
\hline ASP192 & $\bullet$ & 35 & $10 \pm 5$ & $23 \pm 5$ & $12 \pm 10$ & $22 \pm 13$ & 34 & $34 \pm 9$ & $31 \pm 5$ & $21 \pm 8$ & $30 \pm 8$ \\
\hline GLU193 & $\bullet$ & 25 & $53 \pm 14$ & $23 \pm 4$ & $6 \pm 2$ & $45 \pm 15$ & 56 & $46 \pm 6$ & $24 \pm 7$ & $6 \pm 4$ & $41 \pm 9$ \\
\hline GLY194 & $\bullet$ & 6 & 0 & 0 & 0 & $2 \pm 1$ & 27 & $2 \pm 1$ & 0 & 0 & $4 \pm 1$ \\
\hline LEU195 & - & 6 & $14 \pm 6$ & $19 \pm 4$ & $8 \pm 1$ & $15 \pm 6$ & 2 & $18 \pm 6$ & $27 \pm 6$ & $19 \pm 12$ & $26 \pm 11$ \\
\hline ALA196 & $\bullet$ & 42 & $6 \pm 3$ & $18 \pm 9$ & $8 \pm 7$ & $16 \pm 7$ & 31 & $38 \pm 21$ & $36 \pm 8$ & $6 \pm 4$ & $54 \pm 8$ \\
\hline LEU197 & $\bullet$ & 12 & $19 \pm 7$ & $15 \pm 4$ & 0 & $20 \pm 7$ & 37 & $46 \pm 8$ & $11 \pm 4$ & $2 \pm 1$ & $37 \pm 13$ \\
\hline ALA198 & - & 9 & $22 \pm 10$ & $18 \pm 10$ & 0 & $16 \pm 11$ & 25 & $4 \pm 3$ & $2 \pm 1$ & $2 \pm 1$ & $2 \pm 1$ \\
\hline HIS199 & - & 0 & $6 \pm 3$ & $3 \pm 2$ & 0 & $5 \pm 3$ & 35 & $52 \pm 6$ & $46 \pm 6$ & $25 \pm 8$ & $48 \pm 12$ \\
\hline PHE200 & $\bullet$ & 31 & $32 \pm 8$ & $11 \pm 6$ & 0 & $46 \pm 11$ & 2 & $9 \pm 5$ & $7 \pm 4$ & $7 \pm 6$ & $12 \pm 7$ \\
\hline VAL201 & - & 65 & $15 \pm 8$ & $24 \pm 5$ & $4 \pm 3$ & $21 \pm 12$ & 37 & $46 \pm 10$ & $14 \pm 4$ & $2 \pm 1$ & $37 \pm 11$ \\
\hline TRP202 & - & 38 & $43 \pm 8$ & $37 \pm 6$ & $21 \pm 9$ & $39 \pm 6$ & 30 & $25 \pm 3$ & $15 \pm 5$ & $7 \pm 5$ & $15 \pm 5$ \\
\hline ILE203 & - & 10 & $5 \pm 3$ & $20 \pm 7$ & $2 \pm 1$ & $12 \pm 7$ & 60 & $42 \pm 8$ & $26 \pm 13$ & $9 \pm 8$ & $37 \pm 11$ \\
\hline ALA204 & - & 58 & $30 \pm 8$ & $12 \pm 6$ & $4 \pm 3$ & $28 \pm 9$ & 51 & $54 \pm 15$ & $20 \pm 11$ & $4 \pm 3$ & $43 \pm 18$ \\
\hline PRO205 & - & 63 & $55 \pm 6$ & $66 \pm 4$ & $33 \pm 2$ & $62 \pm 10$ & 4 & $14 \pm 5$ & $11 \pm 6$ & $2 \pm 1$ & $27 \pm 10$ \\
\hline LEU206 & - & 46 & $60 \pm 7$ & $36 \pm 8$ & $23 \pm 20$ & $61 \pm 14$ & 64 & $77 \pm 11$ & $72 \pm 6$ & $48 \pm 23$ & $75 \pm 10$ \\
\hline GLN207 & - & 18 & $24 \pm 6$ & $12 \pm 7$ & $9 \pm 7$ & $34 \pm 9$ & 50 & $42 \pm 7$ & $18 \pm 10$ & $6 \pm 2$ & $35 \pm 10$ \\
\hline VAL208 & - & 37 & $14 \pm 5$ & $14 \pm 8$ & $45 \pm 4$ & $14 \pm 11$ & 54 & $34 \pm 8$ & $37 \pm 7$ & $13 \pm 4$ & $55 \pm 9$ \\
\hline ALA209 & - & 61 & $73 \pm 12$ & $67 \pm 10$ & $33 \pm 16$ & $75 \pm 10$ & 48 & $26 \pm 9$ & $16 \pm 6$ & $9 \pm 5$ & $28 \pm 11$ \\
\hline LEU210 & - & 25 & $34 \pm 11$ & $29 \pm 13$ & $24 \pm 23$ & $31 \pm 13$ & 112 & $98 \pm 8$ & $80 \pm 10$ & $58 \pm 14$ & $95 \pm 13$ \\
\hline LEU211 & - & 5 & $6 \pm 3$ & $8 \pm 3$ & $8 \pm 7$ & $15 \pm 7$ & 83 & $64 \pm 7$ & $49 \pm 8$ & $36 \pm 13$ & $67 \pm 9$ \\
\hline MET212 & - & 57 & $36 \pm 9$ & $36 \pm 7$ & $58 \pm 20$ & $25 \pm 14$ & 0 & 0 & 0 & $3 \pm 2$ & $2 \pm 1$ \\
\hline GLY213 & $\bullet$ & 90 & $102 \pm 10$ & $99 \pm 9$ & $89 \pm 13$ & $92 \pm 9$ & 69 & $14 \pm 8$ & $18 \pm 13$ & $12 \pm 7$ & $13 \pm 6$ \\
\hline LEU214 & - & 55 & $49 \pm 11$ & $59 \pm 8$ & $53 \pm 4$ & $54 \pm 13$ & 101 & $83 \pm 9$ & $69 \pm 8$ & $66 \pm 17$ & $83 \pm 8$ \\
\hline ILE215 & $\bullet$ & 2 & $1 \pm 1$ & $2 \pm 1$ & $16 \pm 14$ & $4 \pm 2$ & 43 & $24 \pm 5$ & $22 \pm 5$ & $23 \pm 9$ & $25 \pm 5$ \\
\hline TRP216 & - & 57 & $61 \pm 5$ & $54 \pm 7$ & $78 \pm 8$ & $58 \pm 7$ & 3 & $2 \pm 1$ & $2 \pm 1$ & $2 \pm 1$ & $2 \pm 1$ \\
\hline GLU217 & - & 102 & $109 \pm 5$ & $103 \pm 7$ & $\begin{array}{c}100 \pm \\
15\end{array}$ & $103 \pm 8$ & 88 & $50 \pm 6$ & $63 \pm 12$ & $34 \pm 25$ & $77 \pm 9$ \\
\hline LEU218 & $\bullet$ & 33 & $32 \pm 7$ & $24 \pm 9$ & $19 \pm 11$ & $24 \pm 7$ & 103 & $93 \pm 6$ & $94 \pm 9$ & $91 \pm 5$ & $94 \pm 7$ \\
\hline \multicolumn{2}{|c|}{ \%AGREEMENT } & 45 & 41 & 45 & 31 & 45 & 45 & 41 & 52 & 55 & 38 \\
\hline
\end{tabular}


B.

\begin{tabular}{|c|c|c|c|c|c|c|c|c|c|c|c|}
\hline \multirow[b]{2}{*}{ RESIDUE } & \multirow[b]{2}{*}{ EXP } & \multicolumn{5}{|c|}{ DALTON } & \multicolumn{5}{|c|}{ MORNON } \\
\hline & & $\begin{array}{l}\text { Initial } \\
\text { Model }\end{array}$ & $\begin{array}{c}\text { Control } \\
\text { MD }\end{array}$ & MDFF & $\begin{array}{c}\text { Final } \\
\text { Model }\end{array}$ & $\begin{array}{c}\text { Continuation } \\
\text { MD }\end{array}$ & $\begin{array}{l}\text { Initial } \\
\text { Model }\end{array}$ & $\begin{array}{c}\text { Control } \\
\text { MD }\end{array}$ & MDFF & $\begin{array}{l}\text { Final } \\
\text { Model }\end{array}$ & $\begin{array}{c}\text { Continuation } \\
\text { MD }\end{array}$ \\
\hline GLY330 & - & 59 & $121 \pm 8$ & $119 \pm 9$ & $93 \pm 19$ & $103 \pm 28$ & 44 & $71 \pm 18$ & $29 \pm 6$ & $40 \pm 24$ & $54 \pm 30$ \\
\hline ILE331 & $\bullet$ & 43 & $17 \pm 6$ & $24 \pm 7$ & $30 \pm 4$ & $23 \pm 6$ & 92 & $63 \pm 9$ & $87 \pm 18$ & $84 \pm 20$ & $91 \pm 20$ \\
\hline ILE332 & - & 64 & $83 \pm 5$ & $72 \pm 6$ & $72 \pm 1$ & $77 \pm 9$ & 91 & $96 \pm 10$ & $65 \pm 20$ & $68 \pm 13$ & $74 \pm 19$ \\
\hline LEU333 & $\bullet$ & 49 & $49 \pm 11$ & $65 \pm 10$ & $84 \pm 29$ & $62 \pm 11$ & 24 & $27 \pm 5$ & $52 \pm 20$ & $78 \pm 18$ & $36 \pm 14$ \\
\hline ARG334 & $\bullet$ & 101 & $63 \pm 18$ & $84 \pm 9$ & $82 \pm 16$ & $90 \pm 12$ & 90 & $91 \pm 5$ & $86 \pm 4$ & $73 \pm 3$ & $86 \pm 6$ \\
\hline LYS335 & $\bullet$ & 66 & $49 \pm 6$ & $42 \pm 6$ & $36 \pm 8$ & $48 \pm 7$ & 46 & $63 \pm 9$ & $90 \pm 16$ & $83 \pm 11$ & $74 \pm 8$ \\
\hline ILE336 & $\bullet$ & 6 & $8 \pm 3$ & $11 \pm 7$ & $17 \pm 7$ & $7 \pm 5$ & 6 & $9 \pm 6$ & $7 \pm 4$ & $11 \pm 5$ & $6 \pm 5$ \\
\hline PHE337 & $\bullet$ & 45 & $14 \pm 7$ & $57 \pm 7$ & $51 \pm 17$ & $57 \pm 11$ & 80 & $70 \pm 6$ & $56 \pm 10$ & $51 \pm 21$ & $65 \pm 15$ \\
\hline THR338 & $\bullet$ & 53 & $45 \pm 8$ & $50 \pm 9$ & $34 \pm 6$ & $46 \pm 13$ & 58 & $73 \pm 12$ & $87 \pm 13$ & $64 \pm 31$ & $73 \pm 11$ \\
\hline THR339 & $\bullet$ & 0 & 0 & 0 & $8 \pm 7$ & 0 & 4 & $2 \pm 1$ & $8 \pm 7$ & $7 \pm 4$ & $5 \pm 1$ \\
\hline ILE340 & $\bullet$ & 5 & $2 \pm 1$ & $3 \pm 2$ & $20 \pm 12$ & $2 \pm 1$ & 9 & $12 \pm 5$ & $5 \pm 4$ & $4 \pm 3$ & $4 \pm 3$ \\
\hline SER341 & $\bullet$ & 60 & $17 \pm 7$ & $68 \pm 14$ & $37 \pm 23$ & $57 \pm 16$ & 60 & $84 \pm 16$ & $41 \pm 7$ & $27 \pm 7$ & $67 \pm 16$ \\
\hline PHE342 & $\bullet$ & 27 & $8 \pm 4$ & $35 \pm 6$ & $24 \pm 6$ & $24 \pm 11$ & 52 & $36 \pm 5$ & $32 \pm 4$ & $22 \pm 3$ & $30 \pm 6$ \\
\hline CYS343 & - & 0 & $2 \pm 1$ & $2 \pm 1$ & $8 \pm 7$ & $3 \pm 2$ & 14 & $2 \pm 1$ & $2 \pm 1$ & $10 \pm 7$ & 0 \\
\hline ILE344 & $\bullet$ & 14 & $19 \pm 8$ & $8 \pm 5$ & $15 \pm 13$ & $11 \pm 7$ & 88 & $31 \pm 9$ & $10 \pm 5$ & $17 \pm 4$ & $28 \pm 8$ \\
\hline VAL345 & $\bullet$ & 26 & $28 \pm 9$ & $47 \pm 11$ & $3 \pm 2$ & $73 \pm 13$ & 49 & $81 \pm 6$ & $23 \pm 6$ & $3 \pm 2$ & $78 \pm 15$ \\
\hline LEU346 & - & 15 & $16 \pm 5$ & $3 \pm 2$ & $12 \pm 9$ & $15 \pm 6$ & 28 & $3 \pm 2$ & $3 \pm 2$ & $3 \pm 2$ & $8 \pm 6$ \\
\hline ARG347 & - & 7 & $8 \pm 2$ & $6 \pm 2$ & $9 \pm 8$ & $9 \pm 3$ & 23 & $4 \pm 2$ & $5 \pm 3$ & $32 \pm 10$ & $4 \pm 2$ \\
\hline MET348 & $\bullet$ & 35 & $28 \pm 9$ & $11 \pm 6$ & $6 \pm 1$ & $22 \pm 9$ & 61 & $49 \pm 7$ & $9 \pm 4$ & $8 \pm 6$ & $29 \pm 9$ \\
\hline ALA349 & $\bullet$ & 3 & $54 \pm 21$ & $32 \pm 14$ & $3 \pm 2$ & $58 \pm 16$ & 1 & $21 \pm 10$ & $15 \pm 3$ & $3 \pm 2$ & $35 \pm 13$ \\
\hline VAL350 & - & 5 & $13 \pm 5$ & $8 \pm 4$ & $10 \pm 9$ & $11 \pm 8$ & 10 & $3 \pm 2$ & 0 & 0 & $2 \pm 1$ \\
\hline THR351 & - & 36 & $26 \pm 9$ & $29 \pm 6$ & $15 \pm 7$ & $27 \pm 12$ & 23 & $7 \pm 4$ & $2 \pm 1$ & $3 \pm 2$ & $2 \pm 1$ \\
\hline ARG352 & $\bullet$ & 78 & $32 \pm 9$ & $13 \pm 4$ & $2 \pm 1$ & $22 \pm 8$ & 79 & $27 \pm 4$ & $11 \pm 3$ & $3 \pm 2$ & $22 \pm 5$ \\
\hline GLN353 & $\bullet$ & 60 & $49 \pm 9$ & $11 \pm 4$ & 0 & $42 \pm 13$ & 49 & $41 \pm 12$ & $9 \pm 4$ & 0 & $36 \pm 12$ \\
\hline PHE354 & - & 34 & $21 \pm 2$ & $12 \pm 3$ & $10 \pm 9$ & $21 \pm 6$ & 17 & $14 \pm 3$ & $7 \pm 2$ & $5 \pm 4$ & $8 \pm 3$ \\
\hline PR0355 & $\bullet$ & 4 & $11 \pm 3$ & $10 \pm 3$ & $2 \pm 1$ & $12 \pm 8$ & 0 & $11 \pm 4$ & $5 \pm 3$ & 0 & $13 \pm 6$ \\
\hline TRP356 & $\bullet$ & 59 & $58 \pm 11$ & $19 \pm 5$ & 0 & $53 \pm 13$ & 66 & $30 \pm 8$ & $5 \pm 2$ & 0 & $27 \pm 11$ \\
\hline ALA357 & - & 36 & $5 \pm 3$ & 0 & 0 & $8 \pm 5$ & 3 & $4 \pm 3$ & $6 \pm 2$ & 0 & $7 \pm 4$ \\
\hline VAL358 & - & 14 & $2 \pm 1$ & $3 \pm 2$ & 0 & $5 \pm 3$ & 23 & $22 \pm 6$ & $4 \pm 2$ & $2 \pm 1$ & $6 \pm 4$ \\
\hline GLN359 & - & 31 & $28 \pm 11$ & $12 \pm 4$ & $3 \pm 1$ & $21 \pm 8$ & 19 & $15 \pm 6$ & $22 \pm 9$ & $11 \pm 2$ & $23 \pm 8$ \\
\hline THR360 & $\bullet$ & 12 & $22 \pm 7$ & $23 \pm 4$ & $5 \pm 1$ & $24 \pm 7$ & 17 & $12 \pm 4$ & $16 \pm 4$ & $2 \pm 1$ & $16 \pm 8$ \\
\hline TRP361 & - & 51 & $30 \pm 6$ & $30 \pm 6$ & $12 \pm 1$ & $28 \pm 6$ & 44 & $61 \pm 5$ & $50 \pm 4$ & $32 \pm 4$ & $47 \pm 9$ \\
\hline TYR362 & - & 15 & $16 \pm 5$ & $16 \pm 4$ & $14 \pm 5$ & $14 \pm 8$ & 15 & $12 \pm 4$ & $5 \pm 4$ & $5 \pm 4$ & $10 \pm 7$ \\
\hline ASP363 & $\bullet$ & 13 & $36 \pm 13$ & $29 \pm 5$ & $25 \pm 5$ & $34 \pm 9$ & 20 & $27 \pm 7$ & $18 \pm 4$ & $13 \pm 1$ & $19 \pm 9$ \\
\hline SER364 & - & 6 & $8 \pm 3$ & $3 \pm 2$ & 0 & $6 \pm 3$ & 2 & $17 \pm 6$ & $9 \pm 3$ & $4 \pm 2$ & $10 \pm 4$ \\
\hline LEU365 & - & 13 & $33 \pm 25$ & $8 \pm 5$ & $17 \pm 6$ & $11 \pm 9$ & 23 & $49 \pm 11$ & $45 \pm 8$ & $39 \pm 9$ & $45 \pm 13$ \\
\hline \%AGREE & NT & 53 & 56 & 61 & 61 & 69 & 67 & 72 & 50 & 47 & 69 \\
\hline
\end{tabular}


C.

\begin{tabular}{|c|c|c|c|c|c|c|c|c|c|c|c|}
\hline & & \multicolumn{5}{|c|}{ DALTON } & \multicolumn{5}{|c|}{ MORNON } \\
\hline RESIDUE & EXP & $\begin{array}{l}\text { Initial } \\
\text { Model }\end{array}$ & $\begin{array}{c}\text { Control } \\
\text { MD }\end{array}$ & MDFF & $\begin{array}{c}\text { Final } \\
\text { Model }\end{array}$ & $\begin{array}{c}\text { Continuation } \\
\text { MD }\end{array}$ & $\begin{array}{l}\text { Initial } \\
\text { Model }\end{array}$ & $\begin{array}{c}\text { Control } \\
\text { MD }\end{array}$ & MDFF & $\begin{array}{c}\text { Final } \\
\text { Model }\end{array}$ & $\begin{array}{c}\text { Continuation } \\
\text { MD }\end{array}$ \\
\hline LEU983 & - & 0 & $3 \pm 2$ & $2 \pm 1$ & 0 & $4 \pm 3$ & 1 & $3 \pm 2$ & 0 & 0 & $2 \pm 1$ \\
\hline ASP984 & - & 9 & $50 \pm 6$ & $39 \pm 6$ & $14 \pm 12$ & $39 \pm 11$ & 56 & $44 \pm 8$ & $34 \pm 10$ & $22 \pm 12$ & $31 \pm 8$ \\
\hline ASP985 & - & 62 & $89 \pm 10$ & $47 \pm 8$ & $21 \pm 7$ & $72 \pm 18$ & 71 & $50 \pm 9$ & $32 \pm 5$ & $18 \pm 4$ & $42 \pm 6$ \\
\hline LEU986 & - & 1 & $3 \pm 2$ & $4 \pm 2$ & $3 \pm 2$ & $4 \pm 2$ & 13 & $3 \pm 2$ & $2 \pm 1$ & 0 & $2 \pm 1$ \\
\hline LEU987 & - & 1 & $3 \pm 2$ & $3 \pm 2$ & $2 \pm 1$ & $4 \pm 3$ & 9 & $13 \pm 7$ & $7 \pm 4$ & $4 \pm 3$ & $6 \pm 5$ \\
\hline PRO988 & - & 27 & $45 \pm 5$ & $52 \pm 3$ & $41 \pm 2$ & $44 \pm 10$ & 30 & $41 \pm 13$ & $28 \pm 6$ & $19 \pm 4$ & $26 \pm 10$ \\
\hline LEU989 & - & 56 & $29 \pm 5$ & $17 \pm 4$ & $5 \pm 3$ & $30 \pm 10$ & 26 & $54 \pm 11$ & $46 \pm 9$ & $16 \pm 4$ & $51 \pm 8$ \\
\hline THR990 & $\bullet$ & 22 & $12 \pm 4$ & $9 \pm 4$ & $6 \pm 2$ & $7 \pm 6$ & 33 & $2 \pm 1$ & 0 & $4 \pm 2$ & $2 \pm 1$ \\
\hline ILE991 & - & 20 & $18 \pm 3$ & $19 \pm 4$ & $8 \pm 5$ & $13 \pm 5$ & 16 & $21 \pm 3$ & $14 \pm 4$ & $6 \pm 4$ & $14 \pm 5$ \\
\hline PHE992 & - & 1 & $4 \pm 2$ & $2 \pm 1$ & $3 \pm 1$ & $5 \pm 2$ & 0 & $18 \pm 4$ & $16 \pm 4$ & $15 \pm 4$ & $19 \pm 9$ \\
\hline ASP993 & - & 23 & $41 \pm 12$ & $22 \pm 4$ & $8 \pm 3$ & $36 \pm 9$ & 24 & $37 \pm 11$ & $22 \pm 8$ & $2 \pm 1$ & $31 \pm 8$ \\
\hline PHE994 & - & 24 & $24 \pm 6$ & $12 \pm 4$ & $11 \pm 2$ & $17 \pm 6$ & 31 & $35 \pm 7$ & $28 \pm 3$ & $26 \pm 9$ & $34 \pm 5$ \\
\hline ILE995 & - & 56 & $61 \pm 7$ & $47 \pm 5$ & $31 \pm 6$ & $47 \pm 11$ & 54 & $48 \pm 7$ & $46 \pm 6$ & $37 \pm 5$ & $44 \pm 8$ \\
\hline GLN996 & - & 11 & $4 \pm 3$ & $4 \pm 2$ & 0 & $5 \pm 3$ & 9 & $4 \pm 2$ & $2 \pm 1$ & 0 & $3 \pm 2$ \\
\hline LEU997 & - & 19 & $12 \pm 4$ & $6 \pm 2$ & $2 \pm 1$ & $5 \pm 4$ & 17 & $23 \pm 5$ & $10 \pm 3$ & $4 \pm 2$ & $21 \pm 7$ \\
\hline LEU998 & - & 54 & $58 \pm 12$ & $45 \pm 7$ & $37 \pm 7$ & $60 \pm 12$ & 73 & $74 \pm 7$ & $57 \pm 6$ & $64 \pm 14$ & $71 \pm 9$ \\
\hline LEU999 & - & 56 & $56 \pm 5$ & $67 \pm 6$ & $51 \pm 9$ & $64 \pm 7$ & 64 & $68 \pm 7$ & $64 \pm 7$ & $60 \pm 9$ & $76 \pm 10$ \\
\hline ILE1000 & $\bullet$ & 25 & $9 \pm 3$ & $9 \pm 5$ & $22 \pm 13$ & $10 \pm 6$ & 39 & $14 \pm 4$ & $15 \pm 6$ & $16 \pm 8$ & $17 \pm 5$ \\
\hline VAL1001 & - & 16 & $13 \pm 5$ & $7 \pm 4$ & $2 \pm 1$ & $14 \pm 5$ & 26 & $22 \pm 9$ & $15 \pm 5$ & $17 \pm 3$ & $25 \pm 9$ \\
\hline ILE1002 & - & 88 & $90 \pm 5$ & $84 \pm 8$ & $65 \pm 6$ & $87 \pm 6$ & 86 & $82 \pm 6$ & $74 \pm 5$ & $69 \pm 8$ & $81 \pm 7$ \\
\hline GLY1003 & - & 109 & $94 \pm 12$ & $92 \pm 12$ & $88 \pm 17$ & $93 \pm 14$ & 111 & $95 \pm 11$ & $96 \pm 8$ & $102 \pm 2$ & $97 \pm 9$ \\
\hline ALA1004 & - & 16 & $19 \pm 8$ & $19 \pm 5$ & $25 \pm 11$ & $23 \pm 7$ & 0 & $14 \pm 7$ & $18 \pm 10$ & $23 \pm 8$ & $7 \pm 6$ \\
\hline ILE1005 & - & 30 & $30 \pm 7$ & $20 \pm 4$ & $5 \pm 4$ & $33 \pm 9$ & 56 & $32 \pm 11$ & $22 \pm 5$ & $19 \pm 14$ & $33 \pm 8$ \\
\hline ALA1006 & - & 77 & $86 \pm 6$ & $87 \pm 4$ & $96 \pm 2$ & $87 \pm 5$ & 89 & $87 \pm 4$ & $90 \pm 5$ & $95 \pm 2$ & $88 \pm 6$ \\
\hline VAL1007 & - & 77 & $78 \pm 9$ & $79 \pm 5$ & $88 \pm 4$ & $79 \pm 6$ & 80 & $74 \pm 6$ & $90 \pm 7$ & $93 \pm 7$ & $76 \pm 7$ \\
\hline VAL1008 & $\bullet$ & 13 & $11 \pm 4$ & $10 \pm 2$ & $15 \pm 8$ & $13 \pm 8$ & 2 & $10 \pm 5$ & $10 \pm 3$ & $10 \pm 6$ & $10 \pm 3$ \\
\hline ALA1009 & $\bullet$ & 38 & $35 \pm 8$ & $31 \pm 5$ & $33 \pm 2$ & $32 \pm 8$ & 71 & $42 \pm 8$ & $36 \pm 6$ & $39 \pm 8$ & $40 \pm 7$ \\
\hline VAL1010 & $\bullet$ & 106 & $108 \pm 6$ & $107 \pm 3$ & $113 \pm 4$ & $108 \pm 5$ & 106 & $106 \pm 5$ & $106 \pm 5$ & $108 \pm 2$ & $105 \pm 5$ \\
\hline LEU1011 & - & 30 & $39 \pm 12$ & $37 \pm 6$ & $52 \pm 19$ & $37 \pm 8$ & 26 & $33 \pm 7$ & $54 \pm 18$ & $54 \pm 15$ & $34 \pm 11$ \\
\hline GLN1012 & - & 13 & $16 \pm 8$ & $10 \pm 4$ & $16 \pm 4$ & $11 \pm 7$ & 27 & $28 \pm 10$ & $28 \pm 14$ & $28 \pm 16$ & $26 \pm 16$ \\
\hline \%AGREEN & NT & 53 & 43 & 43 & 47 & 40 & 50 & 43 & 47 & 40 & 47 \\
\hline
\end{tabular}


D.

\begin{tabular}{|c|c|c|c|c|c|c|c|c|c|c|c|}
\hline \multirow[b]{2}{*}{ RESIDUE } & \multirow[b]{2}{*}{ EXP } & \multicolumn{5}{|c|}{ DALTON } & \multicolumn{5}{|c|}{ MORNON } \\
\hline & & $\begin{array}{l}\text { Initial } \\
\text { Model }\end{array}$ & $\begin{array}{c}\text { Control } \\
\text { MD }\end{array}$ & MDFF & $\begin{array}{l}\text { Final } \\
\text { Model }\end{array}$ & $\begin{array}{c}\text { Continuation } \\
\text { MD }\end{array}$ & $\begin{array}{l}\text { Initial } \\
\text { Model }\end{array}$ & $\begin{array}{c}\text { Control } \\
\text { MD }\end{array}$ & MDFF & $\begin{array}{c}\text { Final } \\
\text { Model }\end{array}$ & $\begin{array}{c}\text { Continuation } \\
\text { MD }\end{array}$ \\
\hline GLY1125 & - & 92 & $61 \pm 32$ & $90 \pm 10$ & $115 \pm 24$ & $88 \pm 18$ & 88 & $75 \pm 17$ & $73 \pm 9$ & $84 \pm 4$ & $85 \pm 16$ \\
\hline GLU1126 & - & 3 & $43 \pm 7$ & $35 \pm 5$ & $42 \pm 14$ & $35 \pm 11$ & 49 & $60 \pm 11$ & $68 \pm 11$ & $78 \pm 15$ & $63 \pm 12$ \\
\hline GLY1127 & $\bullet$ & 119 & $130 \pm 5$ & $134 \pm 6$ & $126 \pm 1$ & $127 \pm 8$ & 113 & $\begin{array}{c}109 \pm \\
17\end{array}$ & $97 \pm 16$ & $57 \pm 37$ & $110 \pm 12$ \\
\hline ARG1128 & - & 55 & $52 \pm 9$ & $61 \pm 4$ & $54 \pm 8$ & $57 \pm 6$ & 64 & $80 \pm 8$ & $67 \pm 7$ & $66 \pm 7$ & $78 \pm 14$ \\
\hline VAL1129 & $\bullet$ & 13 & $9 \pm 4$ & $15 \pm 2$ & $24 \pm 4$ & $20 \pm 10$ & 20 & $9 \pm 8$ & $27 \pm 17$ & $33 \pm 26$ & $29 \pm 20$ \\
\hline GLY1130 & - & 77 & $28 \pm 12$ & $93 \pm 6$ & $94 \pm 4$ & $96 \pm 17$ & 86 & $80 \pm 29$ & $122 \pm 8$ & $117 \pm 12$ & $114 \pm 11$ \\
\hline ILE1131 & $\bullet$ & 71 & $46 \pm 6$ & $49 \pm 4$ & $55 \pm 2$ & $50 \pm 10$ & 86 & $69 \pm 14$ & $70 \pm 16$ & $68 \pm 26$ & $77 \pm 10$ \\
\hline ILE1132 & - & 0 & $2 \pm 1$ & $2 \pm 1$ & $7 \pm 6$ & $3 \pm 2$ & 2 & $10 \pm 4$ & $6 \pm 4$ & $5 \pm 4$ & $12 \pm 6$ \\
\hline LEU1133 & - & 3 & $2 \pm 1$ & $56 \pm 5$ & $65 \pm 8$ & $43 \pm 14$ & 1 & $8 \pm 7$ & $50 \pm 9$ & $61 \pm 21$ & $55 \pm 19$ \\
\hline THR1134 & $\bullet$ & 82 & $12 \pm 10$ & $15 \pm 6$ & $21 \pm 16$ & $21 \pm 10$ & 95 & $54 \pm 8$ & $30 \pm 8$ & $22 \pm 8$ & $50 \pm 13$ \\
\hline LEU1135 & $\bullet$ & 37 & $15 \pm 4$ & $17 \pm 4$ & $32 \pm 13$ & $19 \pm 7$ & 33 & $27 \pm 7$ & $31 \pm 7$ & $25 \pm 8$ & $31 \pm 7$ \\
\hline ALA1136 & - & 14 & $16 \pm 9$ & $16 \pm 5$ & $8 \pm 3$ & $14 \pm 8$ & 4 & $20 \pm 8$ & $8 \pm 7$ & $21 \pm 7$ & $21 \pm 10$ \\
\hline MET1137 & $\bullet$ & 18 & $24 \pm 6$ & $47 \pm 15$ & $32 \pm 11$ & $46 \pm 16$ & 60 & $44 \pm 10$ & $32 \pm 7$ & $26 \pm 2$ & $64 \pm 17$ \\
\hline ASN1138 & $\bullet$ & 40 & $21 \pm 8$ & $9 \pm 3$ & $21 \pm 12$ & $17 \pm 8$ & 61 & $38 \pm 11$ & $18 \pm 9$ & $14 \pm 4$ & $30 \pm 9$ \\
\hline ILE1139 & $\bullet$ & 17 & $19 \pm 5$ & $24 \pm 4$ & $30 \pm 16$ & $20 \pm 5$ & 6 & $13 \pm 6$ & $15 \pm 7$ & $32 \pm 14$ & $10 \pm 5$ \\
\hline MET1140 & - & 7 & $12 \pm 3$ & $29 \pm 8$ & $15 \pm 8$ & $20 \pm 9$ & 5 & $8 \pm 4$ & $21 \pm 10$ & $23 \pm 8$ & $14 \pm 9$ \\
\hline SER1141 & $\bullet$ & 54 & $19 \pm 11$ & $12 \pm 6$ & $2 \pm 1$ & $30 \pm 15$ & 78 & $97 \pm 13$ & $24 \pm 13$ & $2 \pm 1$ & $90 \pm 20$ \\
\hline THR1142 & $\bullet$ & 6 & $10 \pm 4$ & $5 \pm 3$ & $14 \pm 9$ & $8 \pm 6$ & 3 & $27 \pm 6$ & $33 \pm 12$ & $16 \pm 3$ & $23 \pm 7$ \\
\hline LEU1143 & - & 23 & $22 \pm 11$ & $8 \pm 3$ & $9 \pm 3$ & $24 \pm 10$ & 29 & $17 \pm 4$ & $16 \pm 4$ & $25 \pm 7$ & $17 \pm 7$ \\
\hline GLN1144 & $\bullet$ & 49 & $34 \pm 9$ & $26 \pm 9$ & $2 \pm 1$ & $43 \pm 10$ & 29 & $27 \pm 10$ & $24 \pm 8$ & $2 \pm 1$ & $49 \pm 15$ \\
\hline TRP1145 & $\bullet$ & 62 & $21 \pm 5$ & $9 \pm 3$ & $3 \pm 2$ & $19 \pm 5$ & 64 & $38 \pm 13$ & $8 \pm 2$ & $2 \pm 1$ & $25 \pm 10$ \\
\hline ALA1146 & - & 5 & $7 \pm 6$ & $9 \pm 5$ & $8 \pm 4$ & $11 \pm 9$ & 5 & $43 \pm 9$ & $34 \pm 6$ & $18 \pm 15$ & $40 \pm 9$ \\
\hline VAL1147 & $\bullet$ & 2 & $14 \pm 5$ & $15 \pm 4$ & $3 \pm 1$ & $15 \pm 7$ & 4 & $7 \pm 4$ & $11 \pm 4$ & $3 \pm 2$ & $6 \pm 5$ \\
\hline ASN1148 & $\bullet$ & 56 & $48 \pm 13$ & $13 \pm 6$ & 0 & $53 \pm 16$ & 63 & $60 \pm 6$ & $5 \pm 3$ & 0 & $60 \pm 20$ \\
\hline SER1149 & $\bullet$ & 26 & $7 \pm 6$ & 0 & 0 & $8 \pm 7$ & 2 & $26 \pm 16$ & $8 \pm 4$ & $2 \pm 1$ & $20 \pm 10$ \\
\hline SER1150 & - & 6 & $9 \pm 4$ & $4 \pm 2$ & 0 & $8 \pm 6$ & 1 & $3 \pm 2$ & $3 \pm 2$ & $6 \pm 5$ & $2 \pm 1$ \\
\hline ILE1151 & - & 3 & $30 \pm 14$ & $8 \pm 4$ & 0 & $30 \pm 14$ & 8 & $33 \pm 6$ & $17 \pm 7$ & $6 \pm 4$ & $29 \pm 6$ \\
\hline ASP1152 & $\bullet$ & 43 & $67 \pm 9$ & $72 \pm 10$ & $6 \pm 5$ & $73 \pm 13$ & 58 & $76 \pm 14$ & $32 \pm 12$ & $5 \pm 2$ & $63 \pm 19$ \\
\hline VAL1153 & - & 26 & $17 \pm 6$ & $3 \pm 2$ & $3 \pm 2$ & $6 \pm 4$ & 20 & $17 \pm 8$ & $10 \pm 4$ & $9 \pm 8$ & $18 \pm 7$ \\
\hline ASP1154 & $\bullet$ & 3 & $8 \pm 5$ & $3 \pm 2$ & $2 \pm 1$ & $7 \pm 5$ & 3 & $21 \pm 6$ & $13 \pm 3$ & $4 \pm 3$ & $25 \pm 11$ \\
\hline SER1155 & - & 26 & $3 \pm 2$ & $2 \pm 1$ & $9 \pm 4$ & $6 \pm 5$ & 21 & $57 \pm 12$ & $39 \pm 12$ & $20 \pm 9$ & $57 \pm 14$ \\
\hline LEU1156 & $\bullet$ & 8 & $18 \pm 5$ & $20 \pm 4$ & $9 \pm 4$ & $23 \pm 7$ & 1 & $10 \pm 9$ & $4 \pm 3$ & 0 & $13 \pm 8$ \\
\hline MET1157 & - & 59 & $27 \pm 7$ & $22 \pm 5$ & $16 \pm 6$ & $19 \pm 10$ & 61 & $48 \pm 9$ & $37 \pm 5$ & $30 \pm 3$ & $38 \pm 4$ \\
\hline ARG1158 & - & 25 & $46 \pm 7$ & $39 \pm 5$ & $55 \pm 7$ & $41 \pm 6$ & 1 & $23 \pm 4$ & $17 \pm 3$ & $13 \pm 3$ & $24 \pm 8$ \\
\hline SER1159 & - & 9 & $14 \pm 5$ & $22 \pm 6$ & $20 \pm 8$ & $27 \pm 11$ & 2 & $13 \pm 6$ & $4 \pm 3$ & 0 & $9 \pm 5$ \\
\hline \%AGREE & & 61 & 51 & 42 & 54 & 52 & 67 & 64 & 52 & 40 & 64 \\
\hline
\end{tabular}


Figure 1. RMSD plots for the trajectories obtained from three MDFF simulations initiated from the Dalton (grey, orange, and cyan) and Mornon (green, yellow, and blue) models with respect to the equilibrated structures. The steep increase at the last time point results from the final optimization step.

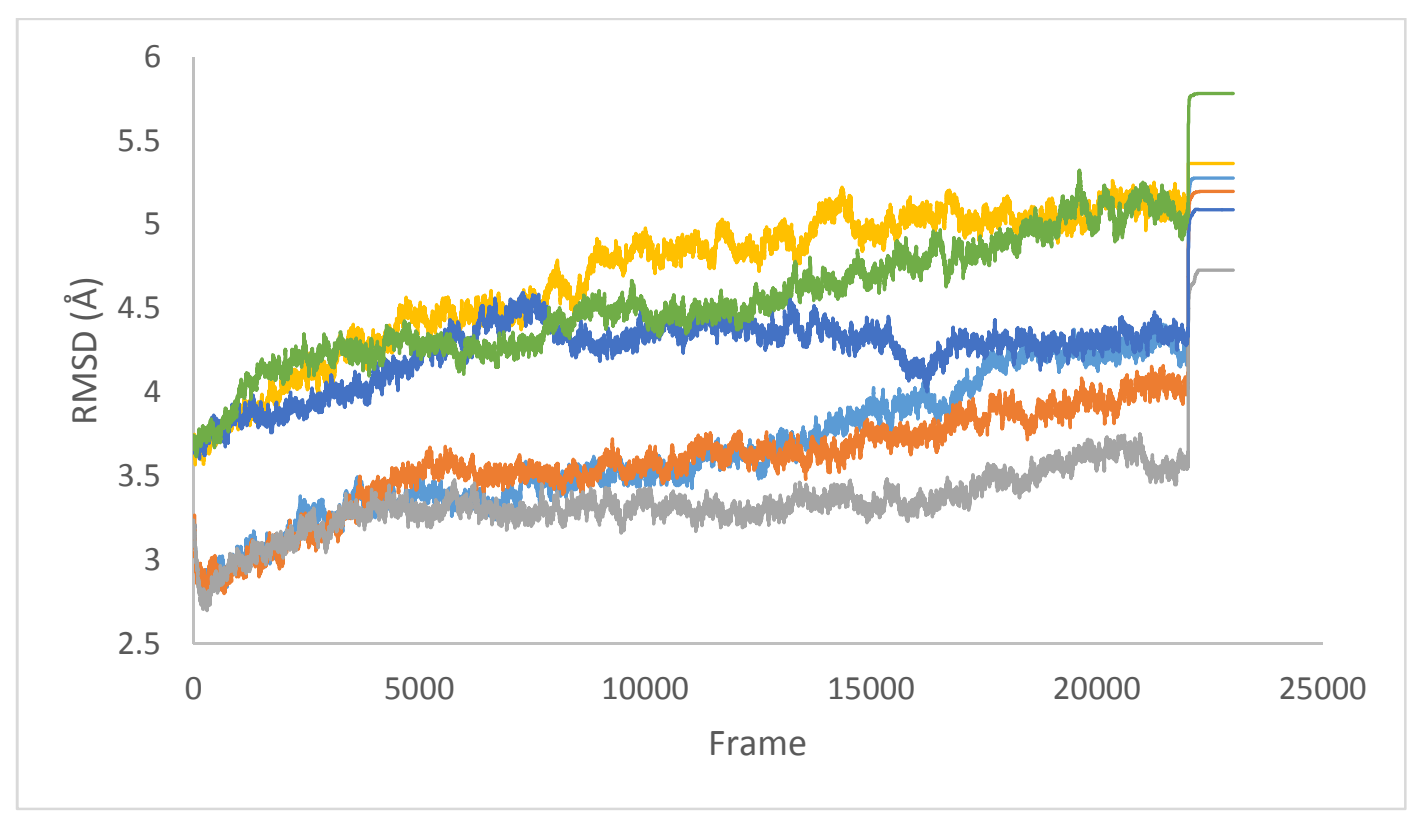


Figure 2. Cross correlation coefficient (CC) plots for the trajectories obtained from three MDFF simulations initiated from the Dalton (grey, orange, and, cyan) and Mornon (green, yellow and blue) models. The steep increase at the last time point results from the final optimization step.

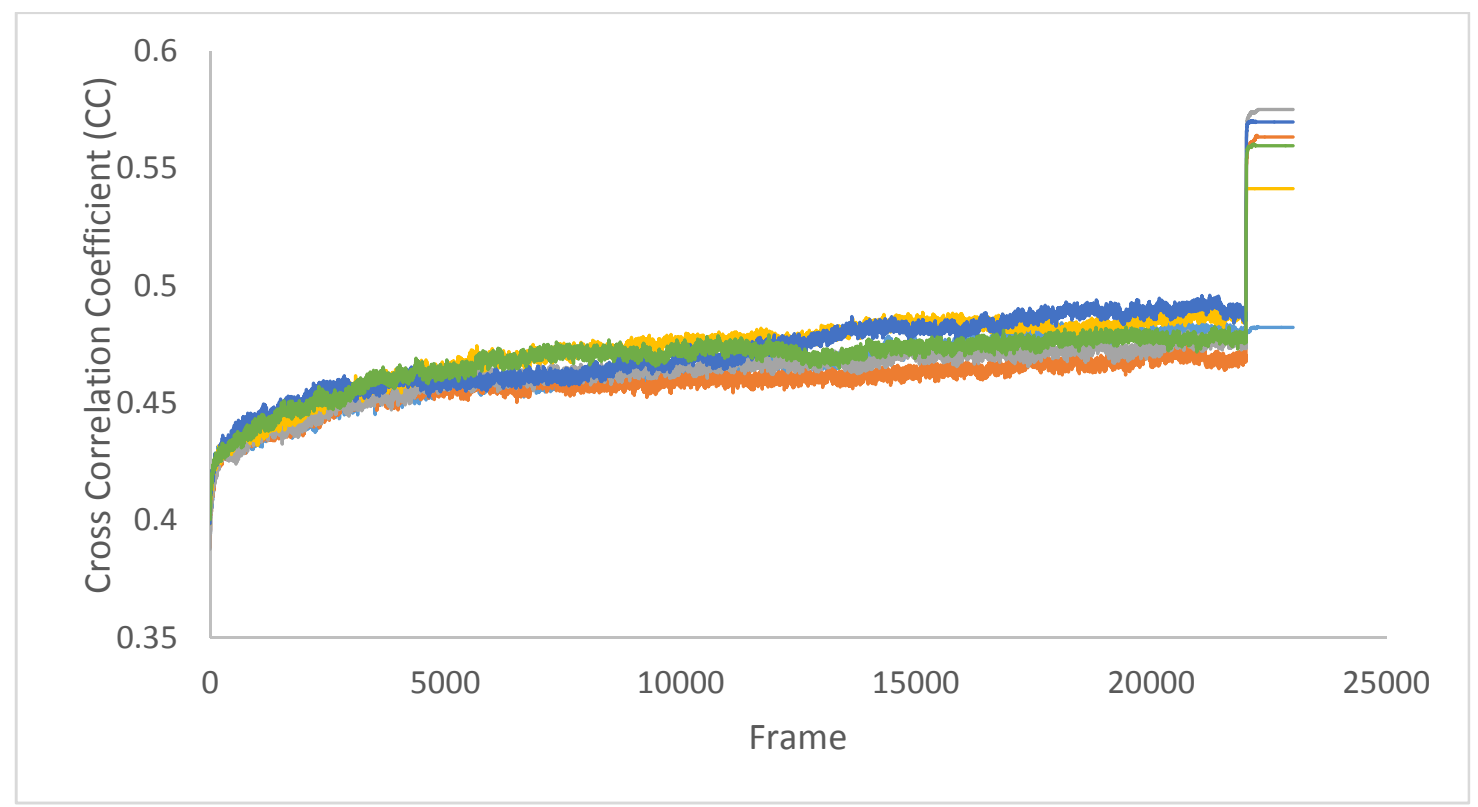


Figure 3. Fitting of the Dalton and Mornon models to the EM map. (A) and (B) present respectively the fitting of the Dalton model prior to and following MDFF. (C) and (D) present respectively the fitting of the Mornon model prior to and following MDFF. Helices 1 and 2 that moved away to the high density region of the map are circled.

A

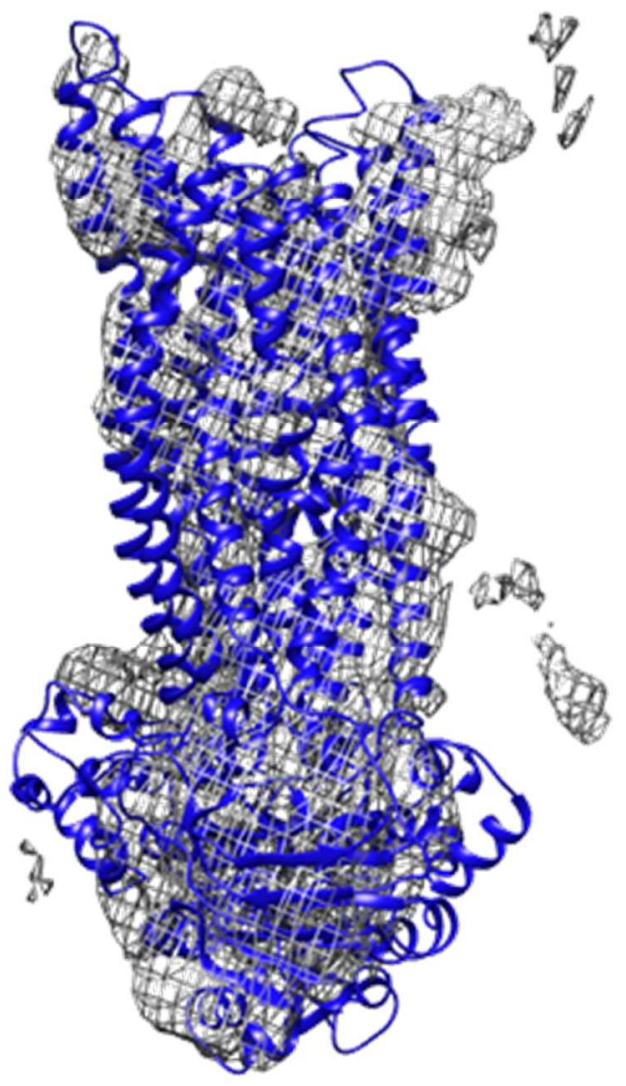

B

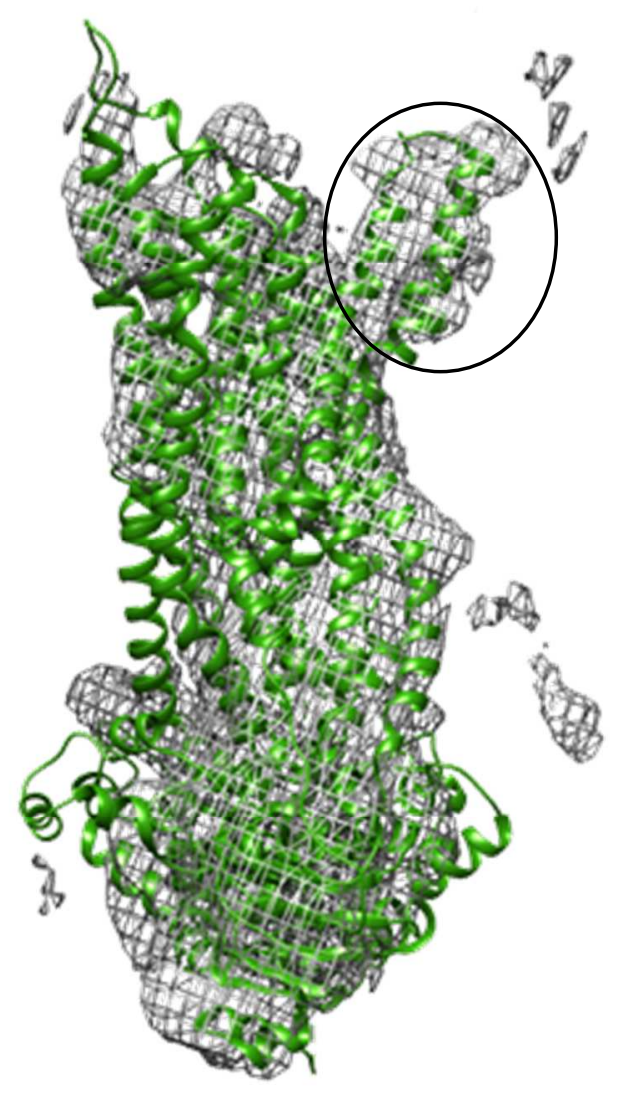


C

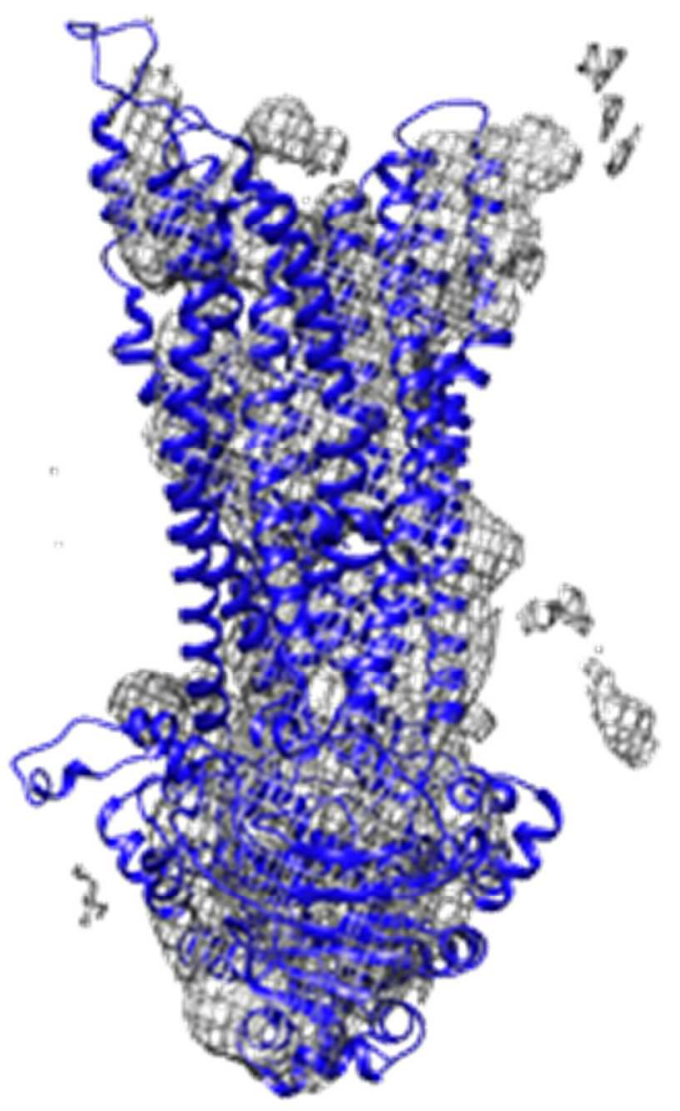

D

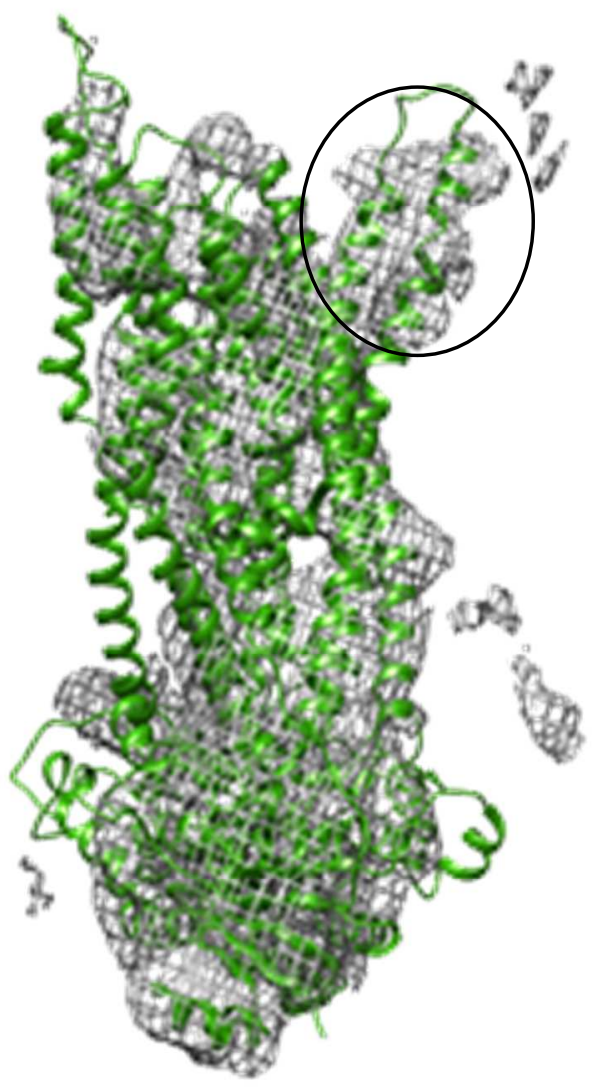


Figure 4. Channel closure upon the movement of helices 6 and 12 of Dalton (A) and Mornon (B) models (blue: before MDFF; purple: after MDFF).

A

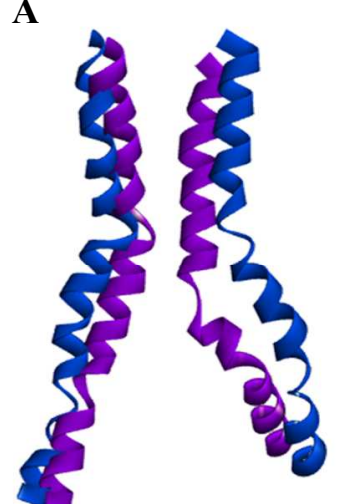

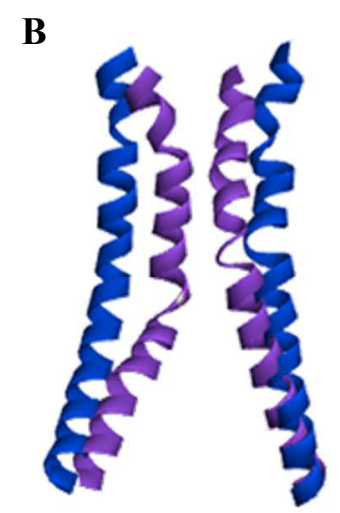


Figure 5. Monitors of salt bridges during MDFF simulations. (A, B): The R347-D924 salt bridge for simulations initiated from the Dalton and Mornon models; (C and D): The K1060-E267 salt bridge for simulations initiated from the Dalton and Mornon models; (E and F): The R352-D993 salt bridge for simulations initiated from the Dalton and Mornon models. Orange, grey, and blue graphs represent individual simulations whereas the yellow graph represents the average of the three simulations. The final jump in the distance values at the last time point is due to the final minimization.

A

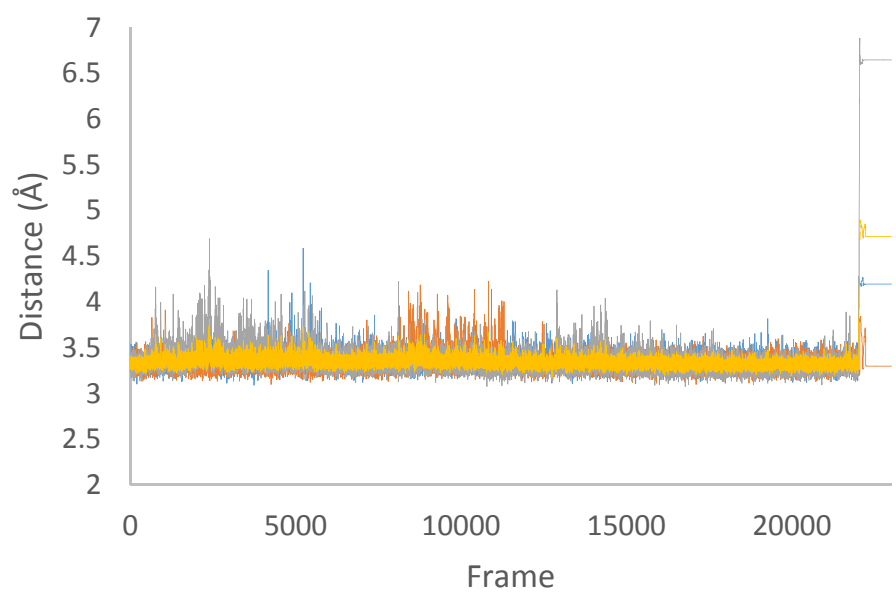

C

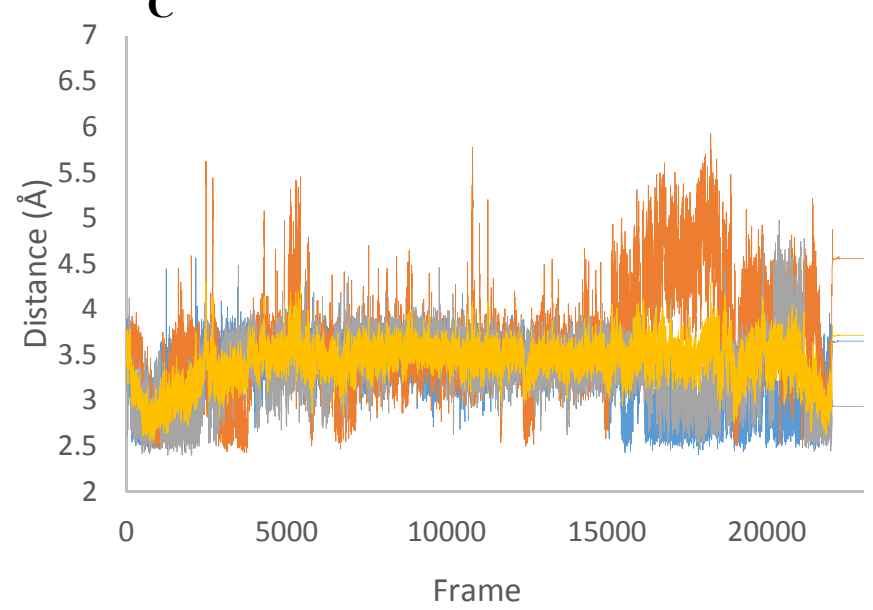

B

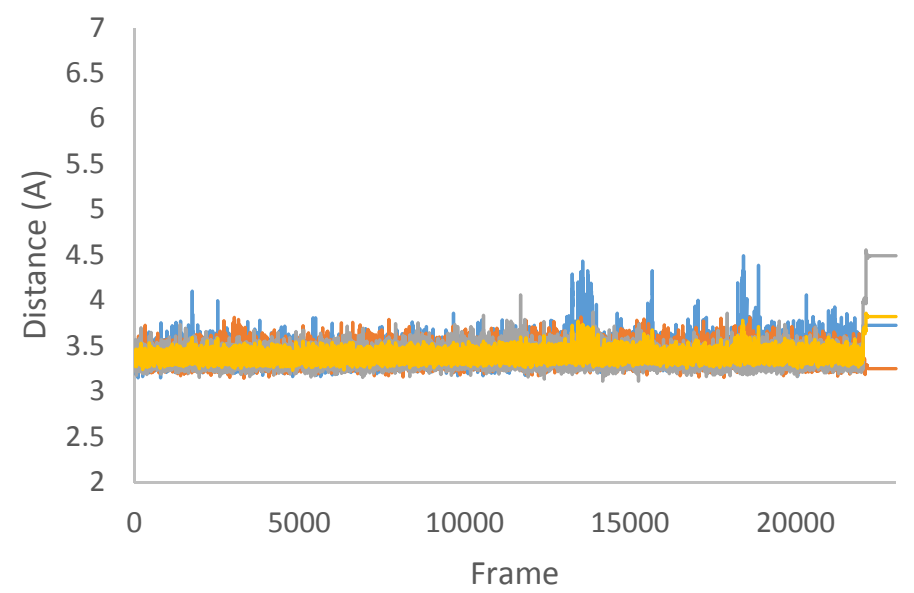

D

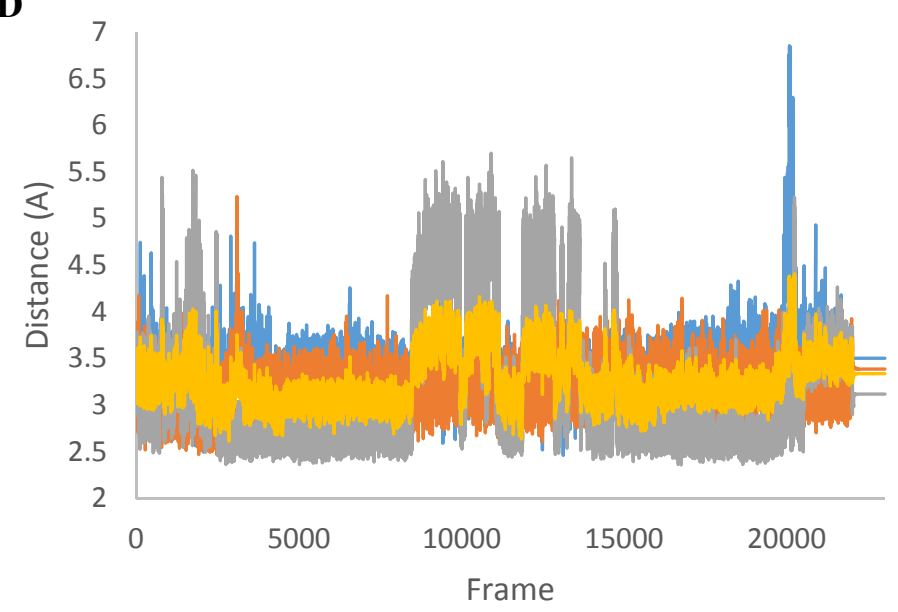



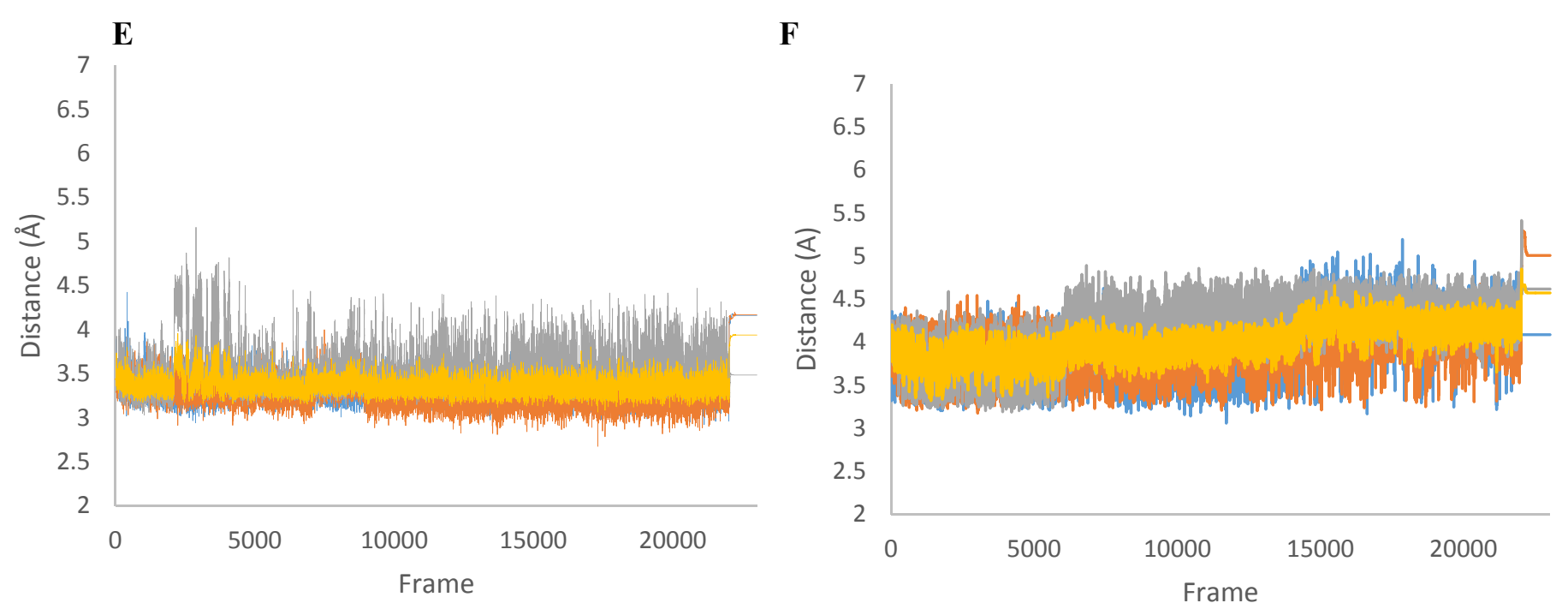

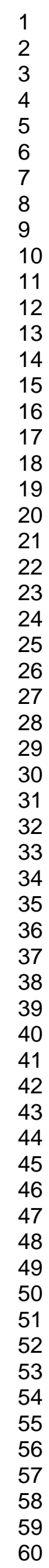

6

7

8

10

11

13

16

17

19

20

22

23

25

26

27

29

30

33

34

35

36

39

40

41

42

43

46

47

48

50

51

52

53

54

55

57

58

59

60 
For Table of Contents Only

1

2

3

4

5

6

7

8

9

10

11

12

13

14

15

16

17

18

19

20

21

22

23

24

25

26

27

28

29

30

31

32

33

34

35

36

37

38

39

40

41

42

43

44

45

46

47

48

49

50

51

52

53

54

55

56

57

58

59

60

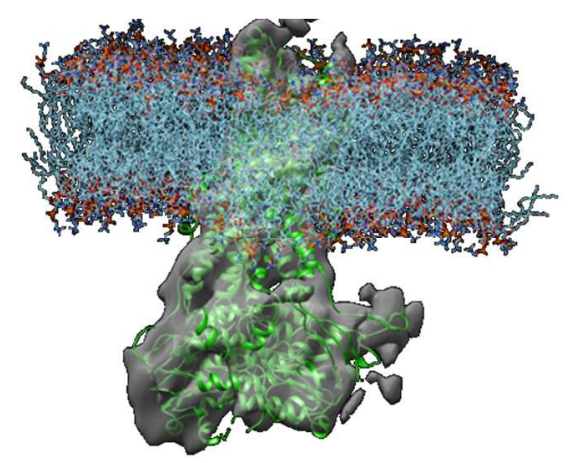


Figure 1. RMSD plots for the trajectories obtained from three MDFF simulations initiated from the Dalton (grey, orange, and cyan) and Mornon (green, yellow, and blue) models with respect to the equilibrated structures. The steep increase at the last time point results from the final optimization step.

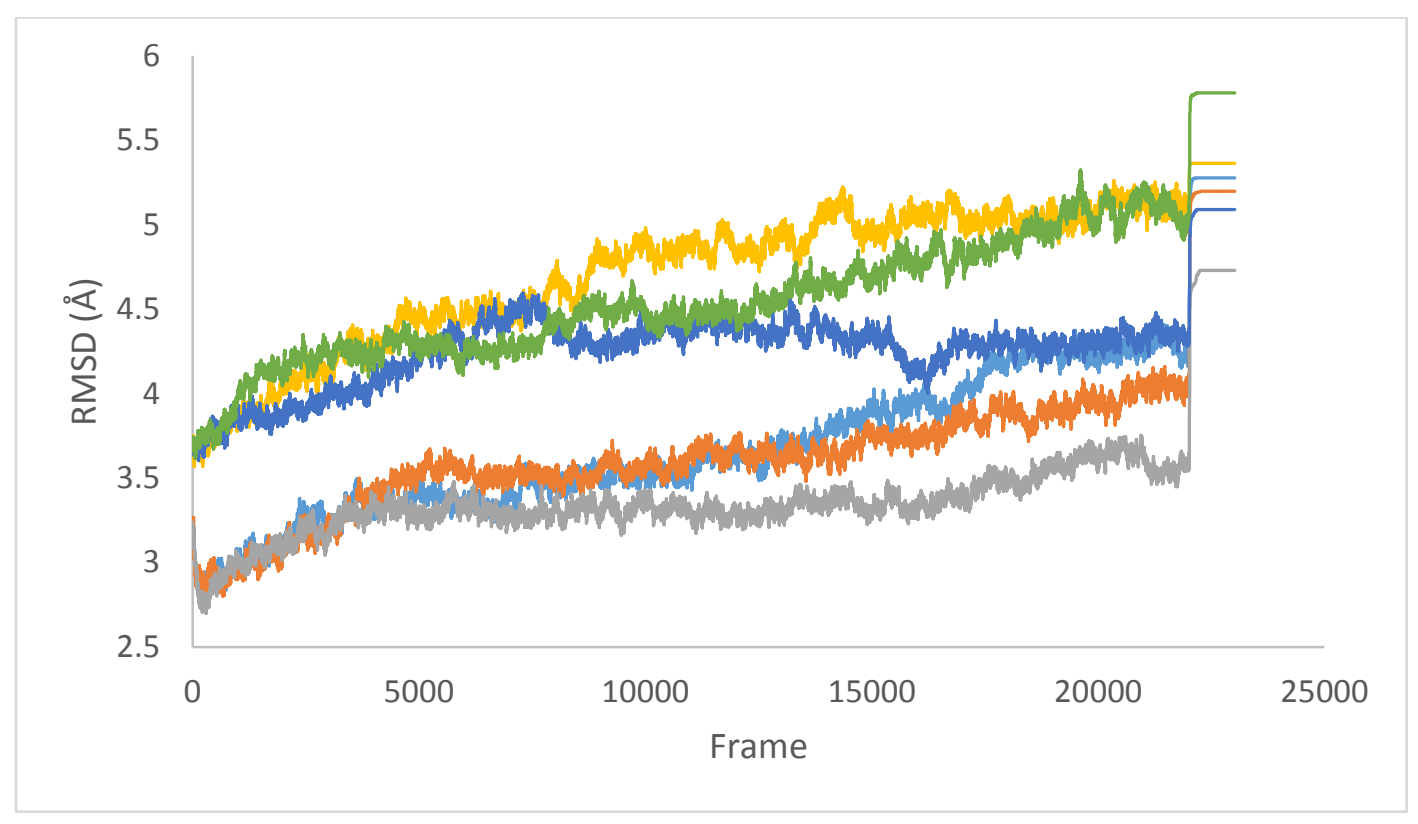


Figure 2. Cross correlation coefficient (CC) plots for the trajectories obtained from three MDFF simulations initiated from the Dalton (grey, orange, and, cyan) and Mornon (green, yellow and blue) models. The steep increase at the last time point results from the final optimization step.

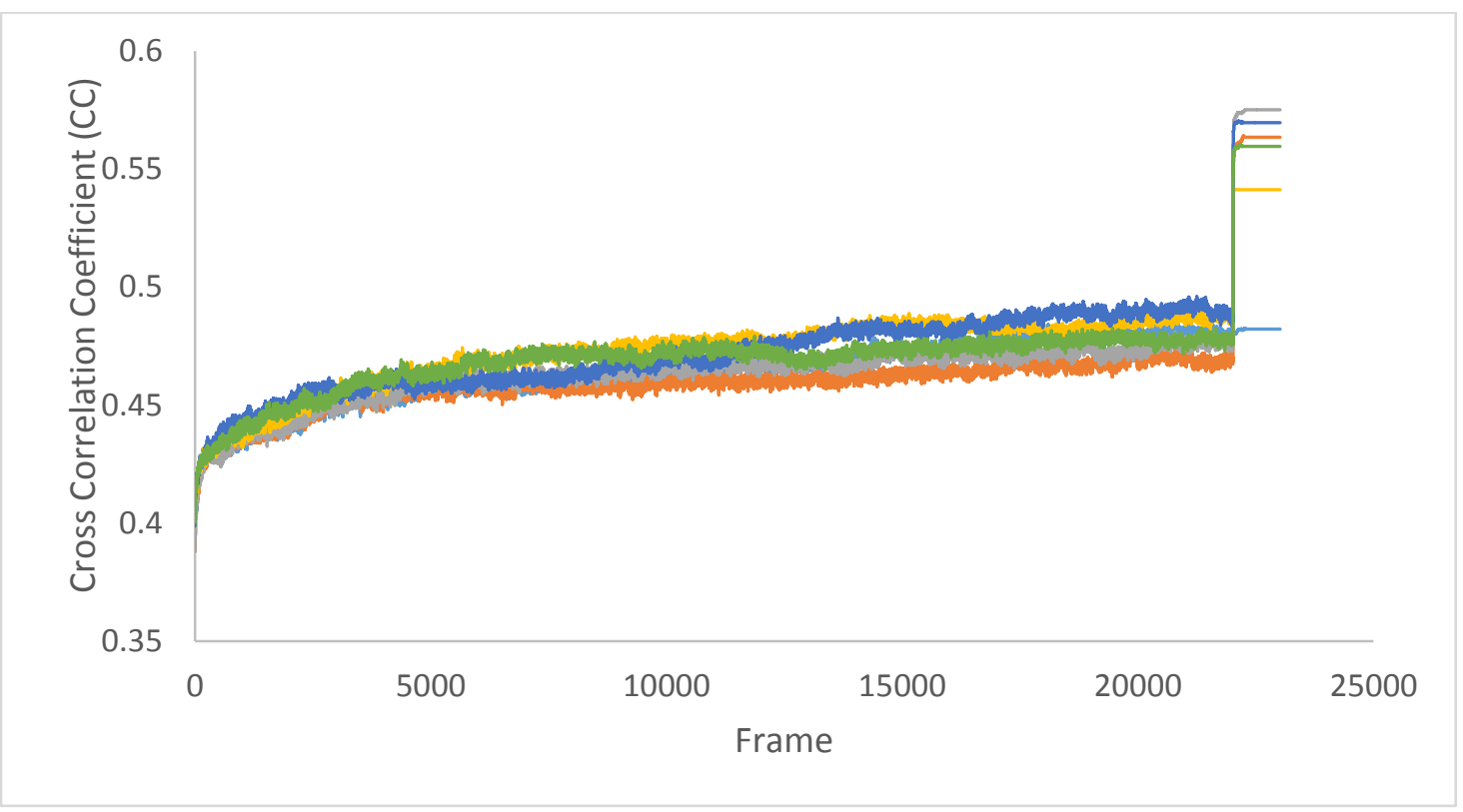


Figure 3. Fitting of the Dalton and Mornon models to the EM map. (A) and (B) present respectively the fitting of the Dalton model prior to and following MDFF. (C) and (D) present respectively the fitting of the Mornon model prior to and following MDFF. Helices 1 and 2 that moved away to the high density region of the map are circled.

A

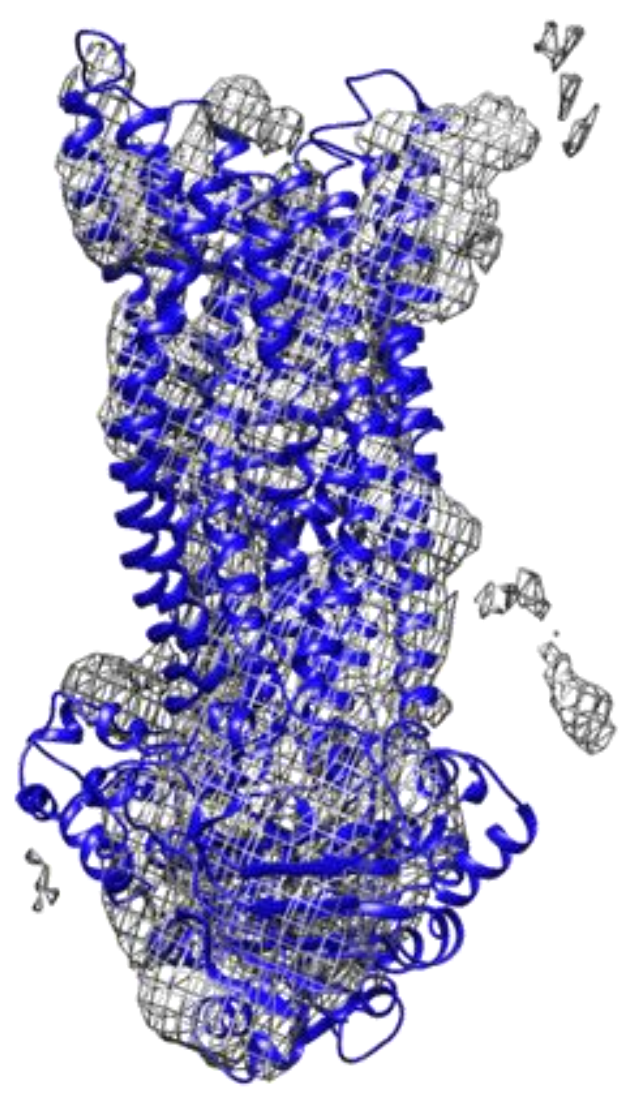

B

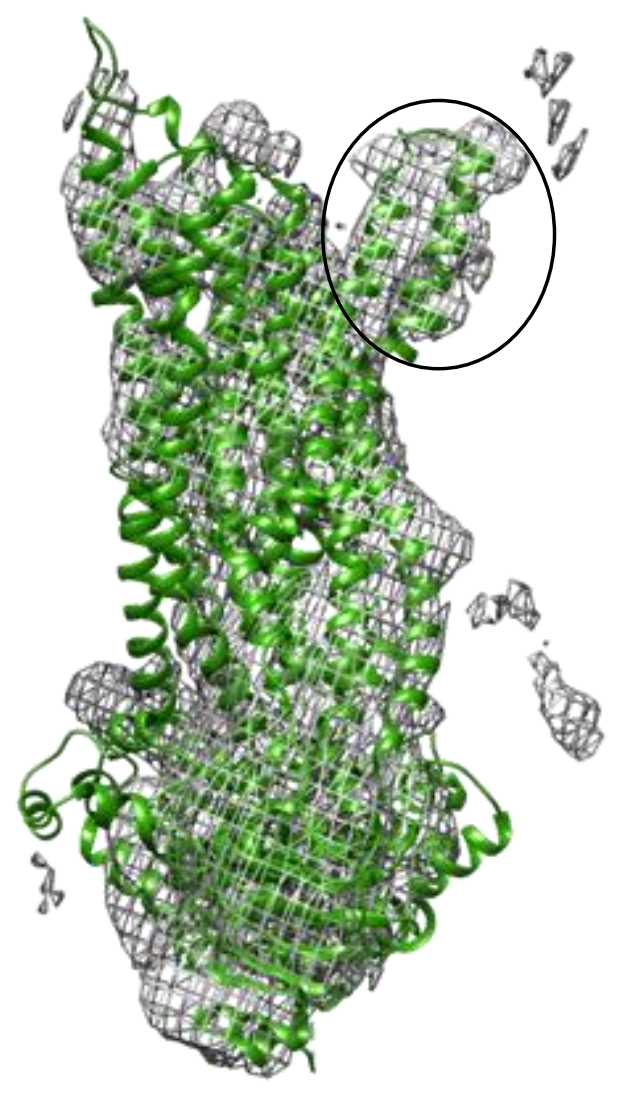


C

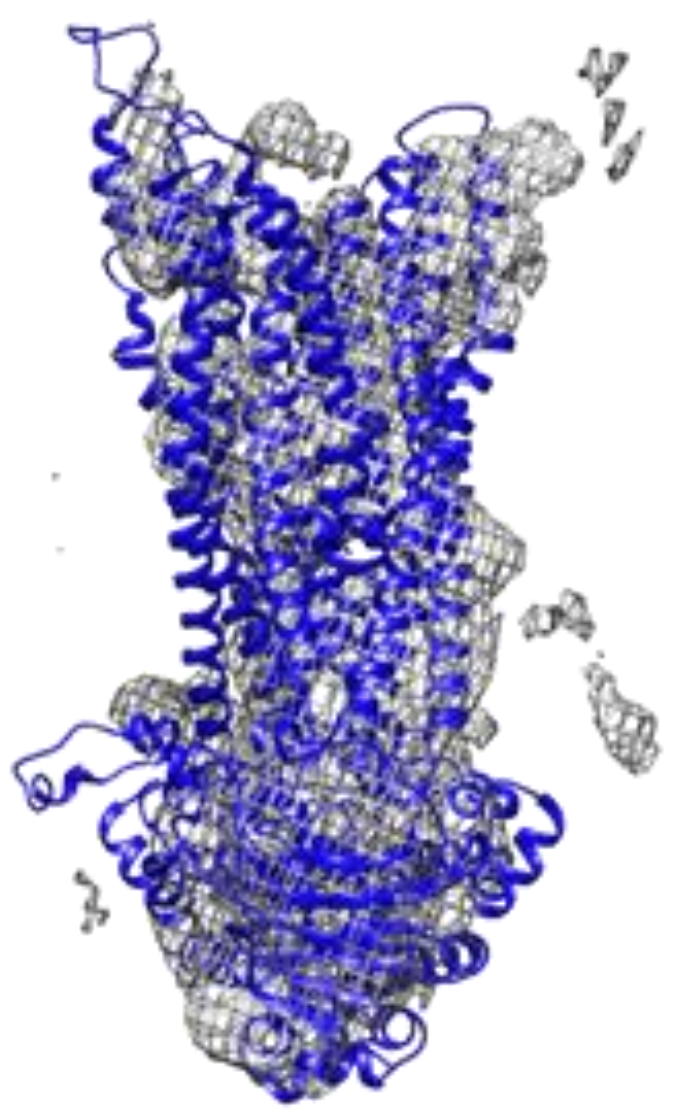

D

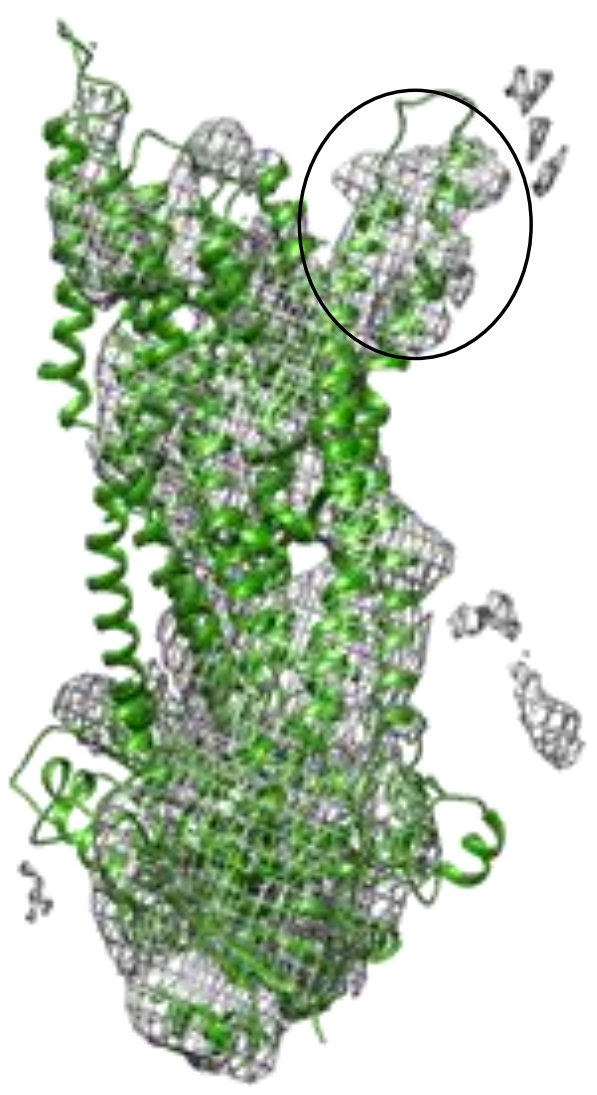


Figure 4. Channel closure upon the movement of helices 6 and 12 of Dalton (A) and Mornon (B) models (blue: before MDFF; purple: after MDFF).

$\mathbf{A}$

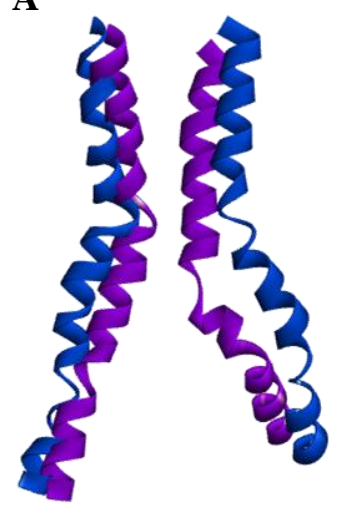

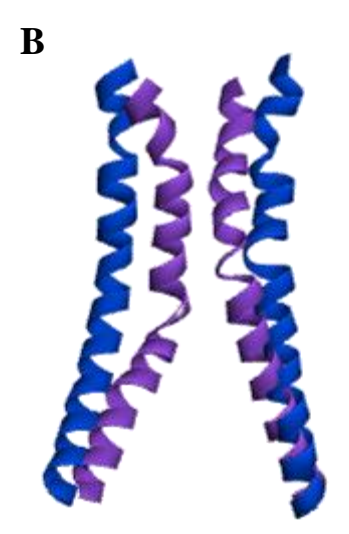


Figure 5. Monitors of salt bridges during MDFF simulations. (A, B): The R347-D924 salt bridge for simulations initiated from the Dalton and Mornon models; (C and D): The K1060-E267 salt bridge for simulations initiated from the Dalton and Mornon models; (E and F): The R352-D993 salt bridge for simulations initiated from the Dalton and Mornon models. Orange, grey, and blue graphs represent individual simulations whereas the yellow graph represents the average of the three simulations. The final jump in the distance values at the last time point is due to the final minimization.

A
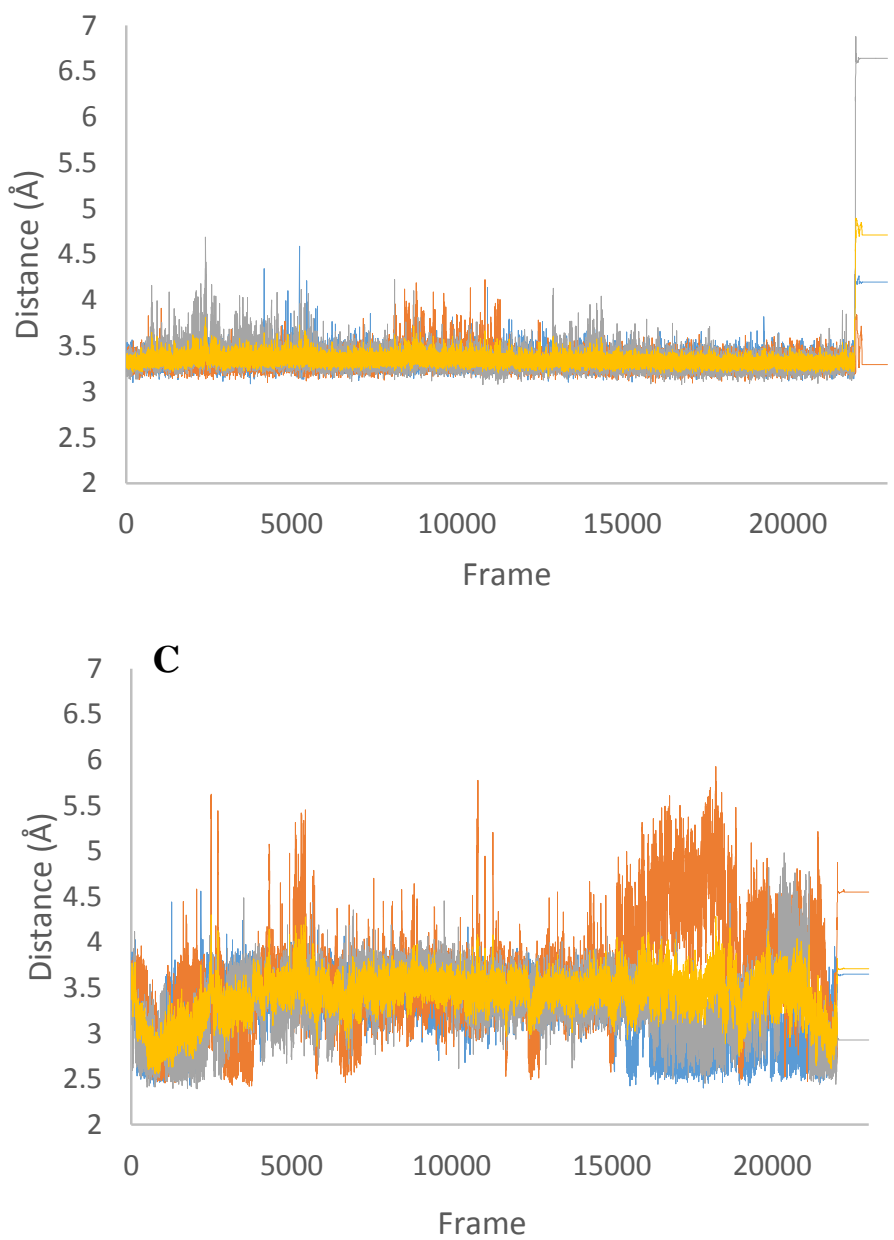

B

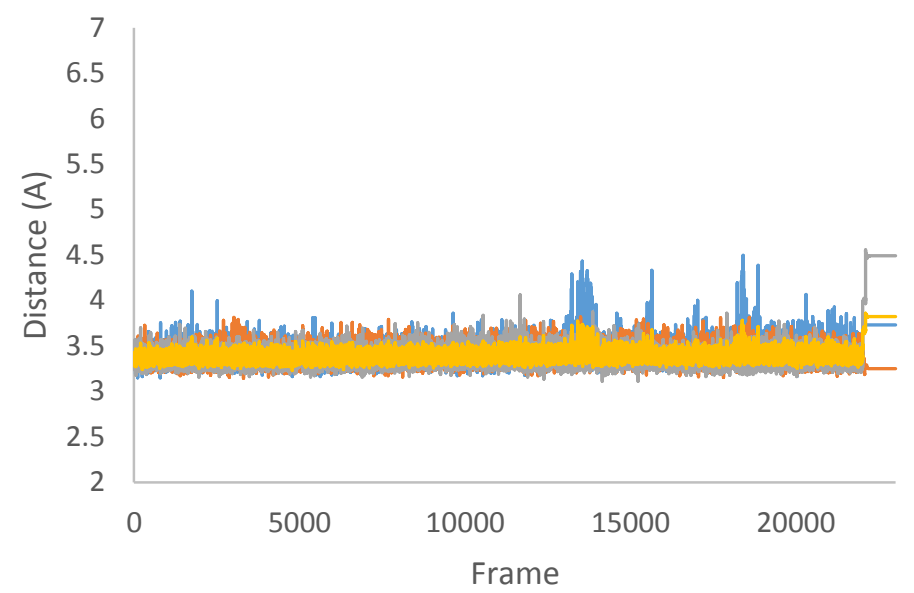

D

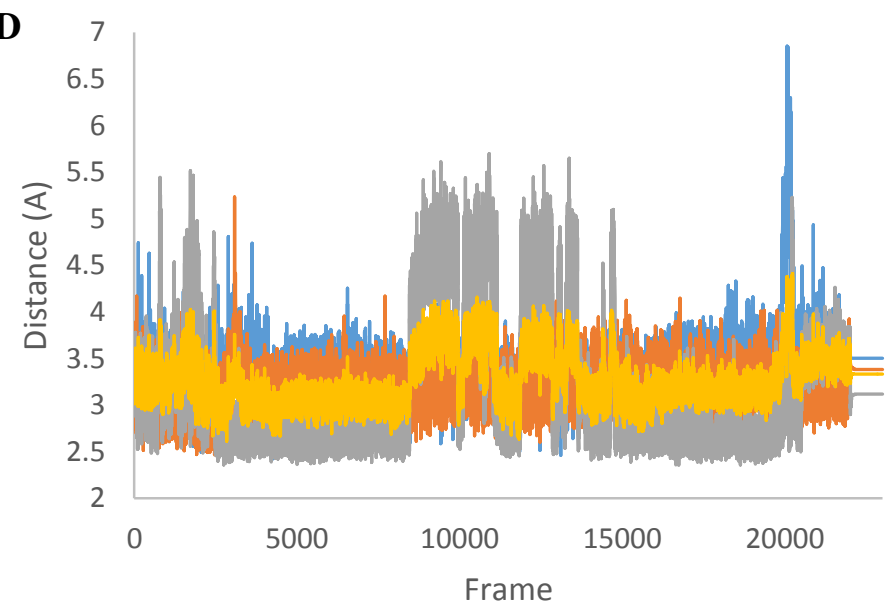




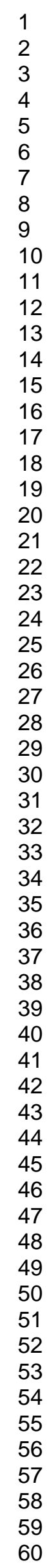
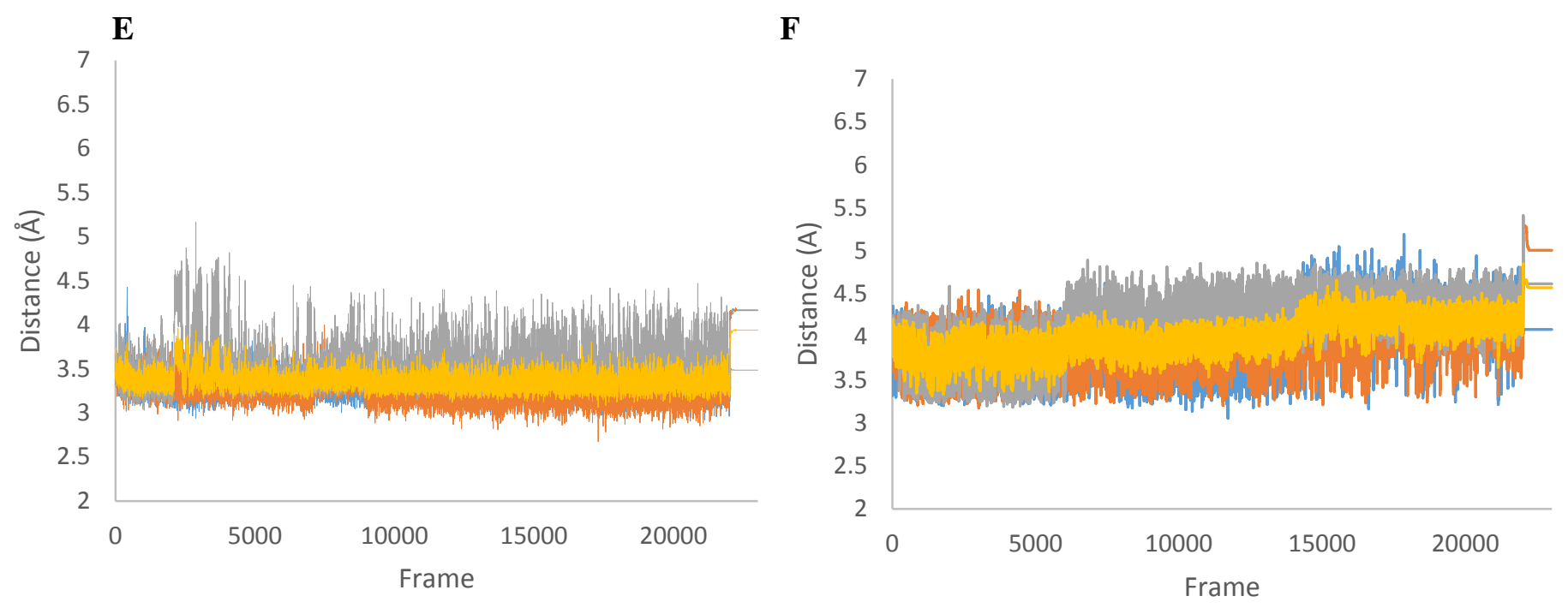

1
2
3
4
5
6
7
8
9
10
11
12
13
14
15
16
17
18
19
20
21
22
23
24
25
26
27
28
29
30
31
32
33
34
35
36
37
38
39
40
41
42
43
44
45
46
47
48
49
50
51
52
53
54
55
56
57
58
60

45 